\title{
Large deviations bound for semiflows over a non-uniformly expanding base
}

\author{
Vítor Araújo (vitor.araujo@im.ufrj.br and vdaraujo@fc.up.pt)* \\ Instituto de Matemática, Universidade Federal do Rio de Janeiro \\ C. P. $68.530,21.945-970$ Rio de Janeiro, RJ-Brazil \\ and \\ Centro de Matemática da Universidade do Porto \\ Rua do Campo Alegre 687, 4169-007 Porto, Portugal.
}

November 6, 2018

Keywords: non-uniform expansion, physical measures, hyperbolic times, large deviations, geometric Lorenz flows, special flows.

2000 Mathematics Subject Classification: Primary: 37D25; Secondary: 37D35, 37D50, $37 \mathrm{C} 40$.

\begin{abstract}
We obtain a exponential large deviation upper bound for continuous observables on suspension semiflows over a non-uniformly expanding base transformation with non-flat singularities or criticalities, where the roof function defining the suspension behaves like the logarithm of the distance to the singular/critical set of the base map. That is, given a continuous function we consider its space average with respect to a physical measure and compare this with the time averages along orbits of the semiflow, showing that the Lebesgue measure of the set of points whose time averages stay away from the space average tends to zero exponentially fast as time goes to infinity.

The arguments need the base transformation to exhibit exponential slow recurrence to the singular set which, in all known examples, implies exponential decay of correlations.

Suspension semiflows model the dynamics of flows admitting cross-sections, where the dynamics of the base is given by the Poincare return map and the roof function is the return time to the cross-section. The results are applicable in particular
\end{abstract}

${ }^{*}$ The author was partially supported by CNPq-Brazil and FCT-Portugal through CMUP and POCI/MAT/61237/2004. 
to semiflows modeling the geometric Lorenz attractors and the Lorenz flow, as well as other semiflows with multidimensional non-uniformly expanding base with nonflat singularities and/or criticalities under slow recurrence rate conditions to this singular/critical set. We are also able to obtain exponentially fast escape rates from subsets without full measure.

\section{Introduction}

The statistical viewpoint on Dynamical Systems provides some of the main tools available for the global study of the asymptotic behavior of transformations or flows. One of the main concepts introduced is the notion of physical (or Sinai-Ruelle-Bowen) measure for a flow or a transformation. An invariant probability measure $\mu$ for a flow $X^{t}$ on a compact manifold is a physical probability measure if the points $z$ satisfying for all continuous functions $\psi$

$$
\lim _{t \rightarrow+\infty} \frac{1}{t} \int_{0}^{t} \psi\left(X^{s}(z)\right) d s=\int \psi d \mu,
$$

form a subset with positive volume (or positive Lebesgue measure) on the ambient space. These time averages are in principle physically observable if the flow models a real world phenomenon admitting some measurable features.

For systems admitting such invariant probability measures it is natural to consider the rate of convergence of the time averages to the space average, given by the volume of the subset of points whose time averages stay away from the space average by a prescribed amount up to some evolution time. This rate is closely related to the so-called thermodynamical formalism first developed for (uniformly) hyperbolic diffeomorphisms, borrowed from statistical mechanics by Bowen, Ruelle and Sinai (among others, see e.g. [22, 23, 51, 52, 29, 21]). These authors systematically studied the construction and properties of physical measures for (uniformly) hyperbolic diffeomorphisms and flows. Such measures for non-uniformly hyperbolic maps and flows where obtained more recently [48, 25, 18, 19, 2].

The probabilistic properties of physical measures are an object of intense study, see e.g. [23, 37, 58, 59, 20, 3, 4, 6, 32, 11, 7]. The main insight behind these efforts is that the family $\left\{\psi \circ X^{t}\right\}_{t>0}$ should behave asymptotically in many respects just like a i.i.d. random variable.

The study of suspension (or special) flows is motivated by modeling a flow admitting a cross-section. Such flow is equivalent to a suspension semiflow over the Poincaré return map to the cross-section with roof function given by the return time function for the points in the cross-section. This is one of the main technical tools in the ergodic theory of Axiom A (or uniformly hyperbolic) flows developed by Bowen and Ruelle [23], enabling them to pass from this type of flow to a suspension flow over a shift transformation with finitely many symbols and bounded roof function. Then the properties of 
the base transformation are used to deduce many results for the suspension flow, which are then passed to the original flow.

Recently, based on the breakthrough of Dolgopyat [27], this kind of modeling provided results on the rate of decay of correlations for certain flows [13] based on the rate of decay of correlations for suspension semiflows [15]. General results on the existence and some statistical properties of physical measures for singular-hyperbolic attractors for three-dimensional flows [10] as well as their sensitive dependence on initial conditions were also obtained using this standard technique. Moreover the classical Lorenz flow [43] was shown to be equivalent to a geometric Lorenz flow by Tucker [54] and so it can be modeled by a suspension semiflow over a non-uniformly hyperbolic transformation with unbounded roof function. Using these ideas it was recently obtained [44] that the physical measure for the Lorenz attractor is mixing.

Here we extend part of the results on large deviation rates of Kifer [37] (see also Waddington [57]) from the uniformly hyperbolic setting to semiflows over non-uniformly expanding base dynamics and unbounded roof function. These special flows model non-hyperbolic flows, like the Lorenz flow, exhibiting equilibria accumulated by regular orbits. We use the properties of non-uniformly expanding transformations, especially the large deviation bound obtained in [7], to deduce a large deviation bound for the suspension semiflow reducing the estimate of the volume of the deviation set to the volume of a certain deviation set for the base transformation. More precisely, if we set $\varepsilon>0$ as an error margin and consider

$$
B_{t}=\left\{z:\left|\frac{1}{t} \int_{0}^{t} \psi\left(X^{t}(z)\right)-\int \psi d \mu\right|>\varepsilon\right\}
$$

then we are able to provide conditions under which the Lebesgue measure of $B_{t}$ decays to zero exponentially fast, i.e. weather there are constants $C, \xi>0$ such that

$$
\operatorname{Leb}\left(B_{t}\right) \leq C e^{-\xi t} \text { for all } t>0 .
$$

The values of $C, \xi>0$ above depend on $\varepsilon, \psi$ and on global invariants for the base transformation $f$, such as the metric entropy and the pressure function of $f$ with respect to the physical measures of $f$ and a certain observable constructed from $\psi$ and $X^{t}$, as detailed in the next section. Having this it is not difficult to deduce exponential escape rates from subsets of the semiflow.

In order to be able to apply this bound to Lorenz flows, it is necessary to allow the roof function of the suspension flows to be unbounded near the singularities of the base dynamical system. This in turn imposes some restrictions on the admissible base dynamics, expressed as a slow recurrence rate to the singular set and uniqueness of equilibrium states with respect to the logarithm of the Jacobian of the map. However no cohomology condition on the roof function are needed, while this is essential to obtain fast decay of correlations in [28, 45, 30].

We present several semiflows with non-uniformly expanding base transformations satisfying all our conditions, including one-dimensional piecewise expanding maps with 
Lorenz-like singularities and quadratic maps but also multidimensional examples. This demanded the detailed study of recurrence rates to the singular set, the study of large deviation bounds for unbounded observables over non-uniformly expanding transformations, and an entropy formula for non-uniformly expanding maps with singularities (which might be of independent interest). Now we give the precise statement of the results.

\subsection{Statement of the results}

Denote by $\|\cdot\|$ a Riemannian norm on the compact boundaryless manifold $M$, by dist the induced distance and by Leb the corresponding Riemannian volume form, which we call Lebesgue measure or volume. We assume Leb to be normalized: $\operatorname{Leb}(M)=1$.

Given a $C^{2}$ local diffeomorphism (Hölder- $C^{1}$ is enough, see below) $f: M \backslash \mathcal{S} \rightarrow M$ outside a volume zero non-flat singular set, let $X^{t}: M_{r} \rightarrow M_{r}$ be a semiflow with roof function $r: M \backslash \mathcal{S} \rightarrow \mathbb{R}$ over the base transformation $f$, as follows. Set $M_{r}=\{(x, y) \in$ $M \times[0,+\infty): 0 \leq y<r(x)\}$. For $x=x_{0} \in M$ denote by $x_{n}$ the $n$th iterate $f^{n}\left(x_{0}\right)$ for $n \geq 0$. Denote $S_{n} \varphi\left(x_{0}\right)=S_{n}^{f} \varphi\left(x_{0}\right)=\sum_{j=0}^{n-1} \varphi\left(x_{j}\right)$ for $n \geq 1$ and for any given real function $\varphi$ in what follows. Then for each pair $\left(x_{0}, s_{0}\right) \in X^{r}$ and $t>0$ there exists a unique $n \geq 1$ such that $S_{n} r\left(x_{0}\right) \leq s_{0}+t<S_{n+1} r\left(x_{0}\right)$ and we define

$$
X^{t}\left(x_{0}, s_{0}\right)=\left(x_{n}, s_{0}+t-S_{n} r\left(x_{0}\right)\right) .
$$

The non-flatness of the singular set $\mathcal{S}$ is an extension to arbitrary dimensions of the notion of non-flat singular set from one-dimensional dynamics [26] and means that $f$ behaves like a power of the distance to the singular set. More precisely there are constants $B>1$ and $0<\beta<1$ for which

(S1) $\frac{1}{B} \operatorname{dist}(x, \mathcal{S})^{\beta} \leq \frac{\|D f(x) v\|}{\|v\|} \leq B \operatorname{dist}(x, \mathcal{S})^{-\beta}$;

$$
\begin{aligned}
& \left|\log \left\|D f(x)^{-1}\right\|-\log \left\|D f(y)^{-1}\right\|\right| \leq B \frac{\operatorname{dist}(x, y)}{\operatorname{dist}(x, \mathcal{S})^{\beta}} \\
& |\log | \operatorname{det} D f(x)^{-1}|-\log | \operatorname{det} D f(y)^{-1}|| \leq B \frac{\operatorname{dist}(x, y)}{\operatorname{dist}(x, \mathcal{S})^{\beta}}
\end{aligned}
$$

for every $x, y \in M \backslash \mathcal{S}$ with $\operatorname{dist}(x, y)<\operatorname{dist}(x, \mathcal{S}) / 2$ and $v \in T_{x} M \backslash\{0\}$. We also assume an extra condition related to the geometry of $\mathcal{S}$. This ensures that the Lebesgue measure of neighborhoods $\mathcal{S}$ is comparable to a power of the distance to $\mathcal{S}$, that is there exists $C_{\kappa}, \kappa>0$ such that for all small $\rho>0$

(S4) $\operatorname{Leb}\{x \in M: \operatorname{dist}(x, \mathcal{S})<\rho\} \leq C_{\kappa} \cdot \rho^{\kappa}$.

The singular set $\mathcal{S}$ contains those points $x$ where $f$ is either not defined, is discontinuous, not differentiable or else $D f(x)$ is non-invertible (that is $\mathcal{S}$ contains the set $\mathcal{C}$ of critical 
points of $f$ ). Note that condition (S4) is satisfied in the particular case when $\mathcal{S}$ is a compact submanifold of $M$, where $\kappa=\operatorname{dim}(M)-\operatorname{dim}(\mathcal{S})$. It is also satisfied for $M=\mathbb{S}^{1}$ and $\mathcal{S}$ is a denumerable infinite subset with finitely many accumulation points, with $\kappa=1$. In particular this holds for a piecewise expanding map over the interval or the circle with finitely many domains of monotonicity.

We say that $f$ is non-uniformly expanding if there exists $c>0$ such that

$$
\limsup _{n \rightarrow+\infty} \frac{1}{n} S_{n} \psi(x) \leq-c \quad \text { where } \quad \psi(x)=\log \left\|D f(x)^{-1}\right\|,
$$

for Lebesgue almost every $x \in M$. This condition implies in particular that all the lower Lyapunov exponents of the map $f$ are strictly positive Lebesgue almost everywhere.

Let $\Delta_{\delta}(x)=\left|\log d_{\delta}(x, \mathcal{S})\right|$ be the smooth $\delta$-truncated logarithmic distance from $x \in M$ to $\delta$, i.e. $\Delta_{\delta}(x)$ is non-negative and continuous away from $\delta$, identically zero $2 \delta$-away from $\mathcal{S}$, and equal to $-\log \operatorname{dist}(x, \mathcal{S})$ when $\operatorname{dist}(x, \mathcal{S}) \leq \delta$.

We say that $f$ has exponentially slow recurrence to the singular set $\mathcal{S}$ if for every $\varepsilon>0$ there exists $\delta>0$ such that

$$
\limsup _{n \rightarrow+\infty} \frac{1}{n} \log \operatorname{Leb}\left\{x \in M: \frac{1}{n} S_{n} \Delta_{\delta}(x)>\varepsilon\right\}<0 .
$$

Condition (1.2) implies that $S_{n} \Delta_{\delta} / n \rightarrow 0$ in measure, i.e. for every $\varepsilon>0$ there exists $\delta>0$ such that

$$
\limsup _{n \rightarrow \infty} \frac{1}{n} S_{n} \Delta_{\delta}(x) \leq \varepsilon
$$

for Lebesgue almost every $x \in M$. We say that a map $f$ satisfying (1.3) has slow recurrence to $\mathcal{S}$.

These notions were presented in [5] and in [5, 1] the following result on existence of finitely many absolutely continuous measures was obtained.

Theorem 1.1. Let $f: M \rightarrow M$ be a $C^{2}$ local diffeomorphism outside a singular set $\mathcal{S}$. Assume that $f$ is non-uniformly expanding with slow recurrence to $\mathcal{S}$. Then there are finitely many ergodic absolutely continuous (in particular physical or Sinai-Ruelle-Bowen) f-invariant probability measures $\mu_{1}, \ldots, \mu_{k}$ whose basins cover the manifold Lebesgue almost everywhere, that is $B\left(\mu_{1}\right) \cup \cdots \cup B\left(\mu_{k}\right)=M, \quad$ Leb - mod 0 . Moreover the support of each measure contains an open disk in $M$.

Here the basin of an invariant probability measure $\mu$ is the subset of points $x \in M$ such that $\lim _{n \rightarrow \infty} \frac{1}{n} \sum_{j=0}^{n-1} \delta_{f j(x)}=\mu$ in the weak topology.

Large deviation bounds are usually related to measure theoretic entropy and to equilibrium states. We denote by $\mathcal{M}_{f}$ the family of all invariant probability measures with respect to $f$. Let $J=|\operatorname{det} D f|$. We say that $\mu \in \mathcal{M}_{f}$ is an equilibrium state with respect to the potential $\log J$ if $h_{\mu}(f)=\mu(\log J)$, that is if $\mu$ satisfies the Entropy Formula. We denote 
by $\mathbb{E}$ the subset of $\mathcal{M}_{f}$ consisting of all equilibrium states for $f$. It is not difficult to see (Section 5 for more details) that each physical measure provided by Theorem 1.1]belongs to $\mathbb{E}$.

Another standing assumption on $f$ is that the set $\mathbb{E}$ is formed by a unique $f$-invariant absolutely continuous probability measure (see Section 2 for sufficient conditions for this to occur and for examples of application).

We denote by $v=\mu \ltimes \operatorname{Leb}^{1}$ the natural $X^{t}$-invariant extension of $\mu$ to $M_{r}$ and by $\lambda$ the natural extension of Leb to $M_{r}$, i.e. $\lambda=\operatorname{Leb} \ltimes \operatorname{Leb}^{1}$, where $\mathrm{Leb}^{1}$ is one-dimensional Lebesgue measure on $\mathbb{R}$ : for any subset $A \subset M_{r}$

$$
v(A)=\frac{1}{\mu(r)} \int d \mu(x) \int_{0}^{r(x)} d s \chi_{A}(x, s) \text { and } \lambda(A)=\frac{1}{\operatorname{Leb}(r)} \int d \operatorname{Leb}(x) \int_{0}^{r(x)} d s \chi_{A}(x, s) .
$$

We say that a function $\varphi: M \backslash \mathcal{S} \rightarrow \mathbb{R}$ has logarithmic growth near $\mathcal{S}$ if there exists $K=K(\varphi)>0$ such that

$$
|\varphi| \chi_{B(\delta, \delta)} \leq K \cdot \Delta_{\delta} \text { for all small enough } \delta>0 .
$$

We also say that $f$ is a regular map if for $E \subset M$ such that $\operatorname{Leb}(E)=0$, then $\operatorname{Leb}\left(f^{-1}(E)\right)=0$.

Theorem A. Let $X^{t}$ be a suspension semiflow over a non-uniformly expanding transformation $f$ on the base $M$ which exhibits exponentially slow recurrence to the singular set, where the roof function $r: M \backslash \mathcal{S} \rightarrow \mathbb{R}$ has logarithmic growth near $\mathcal{S}$. Assume that $f$ is a regular map and that the set $\mathbb{E}$ of equilibrium states is formed by a single measure $\mu$. Let $\psi: M_{r} \rightarrow \mathbb{R}$ be a continuous function. Then

$$
\limsup _{T \rightarrow \infty} \frac{1}{T} \log \lambda\left\{z \in M_{r}:\left|\frac{1}{T} \int_{0}^{T} \psi\left(X^{t}(z)\right) d t-v(\psi)\right|>\varepsilon\right\}<0 .
$$

\subsection{Escape rates}

Let $K \subset M_{r}$ be a compact subset. Given $\varepsilon>0$ we can find an open set $W \supset K$ contained in $M_{r}$ and a continuous bump function $\varphi: M_{r} \rightarrow \mathbb{R}$ such that $\operatorname{Leb}(W \backslash K)<\varepsilon$ with $0 \leq \varphi \leq 1, \varphi \mid K \equiv 1$ and $\varphi \mid(M \backslash W) \equiv 0$. Then we get for $n \geq 1$

$$
\left\{x \in K: X^{t}(x) \in K, 0<t<T\right\} \subset\left\{x \in M: \frac{1}{T} \int_{0}^{T} \varphi\left(X^{t}(x)\right) d t \geq 1\right\}
$$

and so we deduce the following using the estimate from Theorem $\mathrm{A}$,

Corollary B. Let $X^{t}$ be a suspension semiflow over a non-uniformly expanding transformation $f$ on the base $M$ in the same setting as in Theorem Let $K$ be a compact subset of $M_{r}$ such that $v(K)<1$. Then

$$
\limsup _{T \rightarrow+\infty} \frac{1}{T} \log \lambda\left(\left\{x \in K: X^{t}(x) \in K, 0<t<T\right\}\right)<0 .
$$




\subsection{Lorentz and Geometric Lorenz flows}

The Lorenz equations

$$
\dot{x}=10(y-x), \quad \dot{y}=28 x-y-x z, \quad \dot{z}=x y-8 z / 3
$$

were presented by Lorenz [43] in 1963 as a simplified model of convection of the Earth's atmosphere. It turned out that these equations became one of the main models showing the presence of chaotic dynamics in apparently simple systems. More recently Tucker [54,55] with a computer assisted proof showed that equations (1.7) and similar equations with nearby parameters define a geometric Lorenz flow, i.e. a three-dimensional flow $X^{t}$ in $\mathbb{R}^{3}$ with a hyperbolic singularity at the origin admitting a neighborhood $U$ (a trapping region) such that $\overline{X^{t}(U)} \subset U$ for all $t>0$ satisfying:

1. the attracting set $\Lambda=\cap_{t>0} X^{t}(U)$ contains the singularity at 0 ;

2. $\Lambda$ contains a dense orbit;

3. there exists a square $S=[-1,1] \times[-1,1] \times\{1\}$ which is a cross-section for $\Lambda \backslash\{0\}$, that is for every $w \in \Lambda \backslash\{0\}$ there exists $t>0$ such that $X^{t}(w) \in S$;

4. the Poincaré first return map to $S$ given by $R: S \backslash \ell \rightarrow S$ is $C^{2}$ and contracts distances exponentially on the $y$ direction, where $\ell=\{0\} \times[-1,1] \times\{1\}$ is the singular line, so each segment $S \cap\{x=$ const $\}$ is contained in a stable manifold. Moreover in general this one-dimensional and co-dimension one foliation of the cross-section $S$ defines a projection $P$ along leaves which is $C^{1+\alpha}$ for some $\alpha>0$;

5. the one-dimensional map $f:[-1,1] \backslash\{0\} \rightarrow[-1,1]$ obtained from $R$ quotienting out the stable manifolds is a piecewise expanding map with singularities known as Lorenz-like map, which is in the setting of the class of examples detailed in Subsection 2.2;

6. the roof function $\tau(w)$ for $w \in S$ is Lebesgue integrable over $S$ and has logarithmic growth near the singular line $\ell$.

It is well known that the attractor of the geometric Lorenz flows (and the attractor for the Lorenz equations after the results of Tucker already mentioned) supports a unique ergodic physical measure $\mu$ (for more details on this construction see e.g. [56]). Figure 1 gives a visual idea of the geometric Lorenz flow. The reader should consult [33, 34, 50] for proofs of the properties stated above and more details on the construction of such flows. Using $\tau$ as a roof function over the base dynamics given by $R$ we see that the dynamics of a geometric Lorenz flow on $U$ is equivalent to a suspension semiflow over $R$ with roof function $\tau$. In addition the uniform contraction along the leaves of the foliation $\{y=$ const $\}$ together with the uniform expansion of the one-dimensional map $f$ enables us to use Theorem $\mathrm{A}$ to deduce 

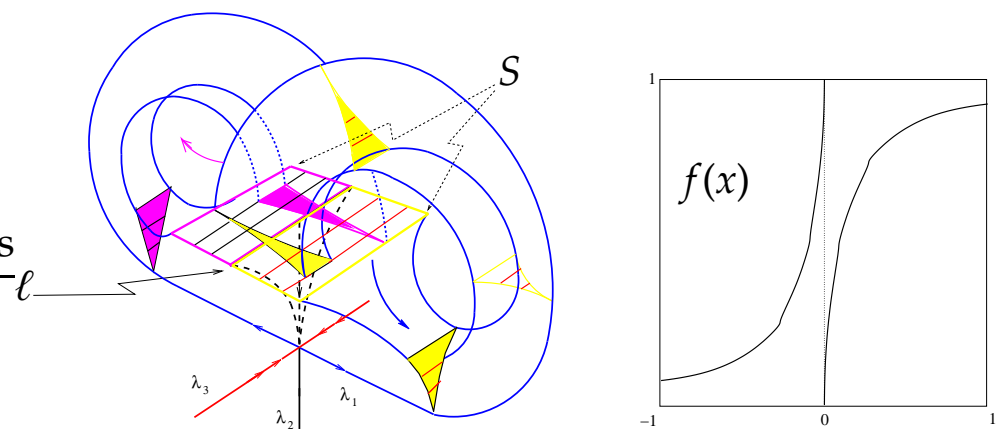

Figure 1: The geometric Lorenz flow and the associated one-dimensional piecewise expanding map

Corollary C. Let $X^{t}$ be a flow on $\mathbb{R}^{3}$ exhibiting a Lorenz or a geometric Lorenz attractor with trapping region $U$. Denoting by Leb the normalized restriction of the Lebesgue volume measure to $U, \psi: U \rightarrow \mathbb{R}$ a bounded continuous function and $\mu$ the unique physical measure for the attractor, then for any given $\varepsilon>0$

$$
\limsup _{T \rightarrow \infty} \frac{1}{T} \log \operatorname{Leb}\left\{z \in U:\left|\frac{1}{T} \int_{0}^{T} \psi\left(X^{t}(z)\right) d t-\mu(\psi)\right|>\varepsilon\right\}<0,
$$

and consequently for any compact $K \subset U$ such that $\mu(K)<1$ we also have

$$
\limsup _{T \rightarrow+\infty} \frac{1}{T} \log \operatorname{Leb}\left(\left\{x \in K: X^{t}(x) \in K, 0<t<T\right\}\right)<0 .
$$

\subsection{Comments and organization of the paper}

We note that the smoothness assumption needed for our arguments is only $C^{1+\alpha}$ for some $\alpha \in(0,1)$. Therefore the $C^{2}$ condition on $f$ in the statements of results can be relaxed to $C^{1+\alpha}$ throughout.

Kifer [37] together with Newhouse [38] obtain sharp large deviations bounds both from above and from below for uniformly partially hyperbolic attractors for flows and for Axiom A flows, through an estimate of the volume growth of images of balls under the action of the flow near the attractor ("volume lemma", see also [23] and [22]). Moreover to obtain the lower bound an assumption of uniqueness of equilibrium states is necessary and this assumption is also used to prove that the upper bound is strictly negative (see also [58] for uniformly expanding transformations and for partially hyperbolic attractors for diffeomorphisms).

Hence the assumption that $\mathbb{E}$ is formed by a single measure is natural in this setting. The author feels this assumption should not be needed to obtain an expression for the upper bound in terms of entropies, as in [37]. However the relevant "volume lemmas" are presently not available in the setting of special flows over non-uniformly expanding base, with singularities or criticalities. Moreover the uniqueness of equilibrium states 
with respect to a large family of potentials (or observables) is still unknown in general (see [47, 12, 11] for recent progress in this direction). Therefore instead of following the approach of [37] we have reduced the problem of estimating the deviations for the suspension flow, with respect to a continuous observable, to the problem of estimating deviations for the base transformation, with respect to an unbounded observable, and then rely on previous work [7] for non-uniformly expanding transformations. To deal with the dynamics near the singularities we impose conditions of very slow recurrence to the singular set $\mathcal{S}$ for the base transformation $f$ together with a growth condition on the roof function $r$ near the singularities. In the end to conclude that the upper bound is strictly negative we use uniqueness of the relevant equilibrium state. Unfortunately this argument does not rule out superexponential decay in (1.5).

Recently Melbourne and Nicol [46] obtained sharp large deviation bounds (i.e. they showed that the limit (1.5) exists) for systems modeled on Markov towers (also known as Young towers) without requiring uniqueness of equilibrium states. In the same work upper large deviation bounds are obtained for semiflows over Markov towers assuming that the roof function is bounded. However their method presents two disadvantages: the large deviation estimates in [46] are proved only for Hölder observables, and these estimates are obtained for the invariant physical measure rather than the volume or Lebesgue measure, which is more directly accessible.

Section 2 shows how the conditions of $f$ and on $r$ are satisfied by many relevant examples. In particular in Subsection 2.4 it is explained how to obtain a large deviation bound for geometric Lorenz flows using the statement of the Main Theorem applied to suspensions semiflows over piecewise expanding maps with singularities, which are treated in a preliminary fashion in Subsection 2.2 and at length in Section 6, The main result needed for the proof of the Main Theorem is a large deviation bound for observables with logarithmic growth near the singular set for a non-uniformly expanding map, which is proved in Section 3. Then the statement of the Main Theorem about large deviations for a suspension semiflow is reduced to a statement of large deviations for the dynamics of the base transformation in Section 4 concluding the proof of the Main Theorem. Note that in contrast to the results on decay of correlations for Anosov flows or Axiom A flows, here we do not need any coboundary conditions on the roof function for the large deviation bound to hold.

In Section 5] we present a derivation of the Entropy Formula for non-uniformly expanding maps with slow recurrence to the singular set, which is used to establish that some examples presented in Section 2 do satisfy our assumptions and which might be interesting in itself.

\section{Acknowledgments}

This paper answers a question posed to me by M. Viana. I am thankful to S. Senti for having pointed to me how to make the example in Subsection 2.3 work. The author is indebted to the openness and hospitality of IMPA, and to its superb library, where most 
of this work was written. I wish to thank the referee for his/her valuable suggestions which helped improve the presentation.

\section{Examples of application}

Here we present some concrete examples where our results can be applied.

\subsection{Suspension semiflows over multidimensional volume expanding and quasi-expanding maps}

Let $f: M \backslash \mathcal{S} \rightarrow M$ be a transitive non-uniformly expanding map with exponentially slow recurrence to $\mathcal{S}$ satisfying $J=|\operatorname{det} D f|>1, \psi=\log \left\|(D f)^{-1}\right\| \leq 0$ and $\psi=0$ at finitely many points only (a quasi-expanding map). We claim that in this setting $\mathbb{E}$ is a singleton.

Indeed $\mathbb{E}$ is non-empty by Theorem 1.1 since every absolutely continuous invariant probability measure is an equilibrium state (see e.g. Theorem 5.1 in Section 5). Since $f \mid M \backslash \mathcal{S}$ is a local diffeomorphism and the support of such absolutely continuous invariant measures contains open sets, the transitivity together with regularity of the map ensure that there exists only one absolutely continuous invariant measure. For otherwise let $\mu_{i}$ be ergodic absolutely continuous $f$-invariant probability measures and let $B_{i} \subset \operatorname{supp}\left(\mu_{i}\right)$ be open sets in the support $i=1,2$; by transitivity and continuity there exists a non-empty open subset $B \subset B_{1}$ and an iterate such that $f^{n}(B) \subset B_{2}$ and by smoothness Leb-almost every point in $f^{n}(B)$ is both a $\mu_{1}$-generic point and a $\mu_{2}-$ generic point, thus $\mu_{1} \equiv \mu_{2}$. This shows that there exists a unique absolutely continuous invariant probability measure for $f$.

Note now that every equilibrium state $v \in \mathbb{E}$ must be such that $h_{v}(f)=v(\log J)>0$ and since $\psi \leq 0$ and has at most finitely many zeroes, then either $v(\psi)<0$ and by Theorem 5.1 the measure $v$ must be absolutely continuous, or $v(\psi)=0$ and $\operatorname{supp} v \subseteq \psi^{-1}(\{0\})$ is finite thus $h_{v}(f)=0$, a contradiction.

Therefore by the uniqueness result above $v$ must coincide with $\mu$. We have shown that $\mathbb{E}=\{\mu\}$, as claimed.

Hence we can apply Theorem A for semiflows over non-uniformly expanding maps with exponentially slow recurrence to the singular set which are also transitive, volume expanding and expanding except at finitely many points, and whose roof function grows with the logarithm of the distance to $\mathcal{S}$.

For examples of multidimensional local diffeomorphisms in this setting see [9]. In this case $\mathcal{S}=\emptyset$ and we can apply Theorem $\mathrm{A}$ for semiflows with this type of base transformation plus a continuous (and thus bounded) roof function.

Clearly the same large deviation bound holds for a semiflow over a local diffeomorphisms which is uniformly expanding together with any continuous roof function. 


\subsection{Suspension semiflows over piecewise expanding maps with sin- gularities}

Let $M$ be the circle $\mathbb{S}^{1}$ or the interval $[0,1]$ with $\{0,1\} \subset \mathcal{S}$ and $\mathcal{S} \subset M$ an at most denumerable and non-flat singular set of $f$ such that its closure $\overline{\mathcal{S}}$ has zero Lebesgue measure: $\operatorname{Leb}(\overline{\mathcal{S}})=0$.

If we assume that $-\infty<\psi<-c<0$ on $M \backslash \mathcal{S}$ for some $c>0$ (so that in particular there are no critical points: $\mathcal{C}=\emptyset$ ) and that $f$ is transitive with slow recurrence to $\mathcal{S}$, then the set $\mathbb{E}$ of equilibrium states with respect to $\log \left|f^{\prime}\right|$ is formed by a single absolutely continuous invariant probability measure, as shown in Subsection 2.1, since $f$ is automatically non-uniformly expanding, quasi-expanding and volume expanding as well.

Observe that for $C^{2}$ maps in our conditions with finitely many smoothness domains, or with derivative of bounded variation, it is well known that there exists a unique ergodic absolutely continuous invariant probability measure $\mu$ with bounded density [35, 53]. Since the function $\log \operatorname{dist}(x, \mathcal{S})$ is Leb-integrable we also have that this function is $\mu$-integrable. Thus for all $\varepsilon>0$ there is $\delta>0$ such that $\int\left|\log \operatorname{dist}_{\delta}(x, \delta)\right| d \mu(x)<\varepsilon$. By the ergodicity and absolute continuity of $\mu$ this means that $f$ has slow recurrence to $\delta$ for a positive Lebesgue measure subset of $M$. Theorem 1.1 together with [5] ensure that $f$ is in fact non-uniformly expanding with slow recurrence to $\mathcal{S}$. Moreover by [36] the same argument applies to $C^{1+\alpha}$ piecewise expanding maps with finitely many smoothness domains, for some $\alpha \in(0,1)$.

To be able to apply the Main Theorem we need exponentially slow recurrence to $\mathcal{S}$. We prove this in Section 6 assuming that $\left|f^{\prime}\right|$ grows as the inverse of some power of the distance to $\mathcal{S}^{\prime}=\mathcal{S} \cap f(M)$, i.e. besides conditions (S1) through (S4) we impose

$$
\left|f^{\prime}(x)\right| \geq B^{-1} \operatorname{dist}\left(x, \mathcal{S}^{\prime}\right)^{-\beta} \text { for all } x \in M \backslash \mathcal{S},
$$

where $\mathcal{S}^{\prime}$ is the (sub)set of singularities which matters for the asymptotic dynamics of $f$.

Hence a semiflow over a piecewise expanding map with singularities satisfying some technical conditions, and with a roof function having logarithmic growth near the singularities admits a large deviation bound as in Theorem $\mathrm{A}$.

\subsection{Suspension semiflows over quadratic maps on Benedicks-Carleson parameters}

Set $M=I=[-1,1]$ and suppose the transformation $f$ is given by $f_{a}(x)=a-x^{2}$ for $a \in\left[a_{0}, 2\right]$ in the positive Lebesgue measure subset constructed by Benedicks and Carleson in [16, 17], where $a_{0} \approx 2$. The properties of the family $f_{a}$ have been thoroughly studied by a considerable number of people. We just mention that Freitas in [31] showed that for these parameters $f_{a}$ is not only a non-uniformly expanding map with $\mathcal{S}=\mathcal{C}=\{0\}$ but also exhibits exponentially slow approximation to the singular set. Actually in [31] only subexponentially slow approximation is stated but the same arguments yield an 
exponential bound as well, as obtained in a much more delicate setting with infinitely many critical points in [8].

Moreover Bruin and Keller [24] show that for this class of maps (specifically for Collet-Eckman maps, i.e. such that $\liminf _{n \rightarrow \infty}\left|\left(f_{a}^{n}\right)^{\prime}(a)\right|^{1 / n}>1$ without extra conditions of recurrence to the criticality) the unique absolutely continuous invariant probability measure is also the unique equilibrium state with respect to $\log \left|f_{a}^{\prime}\right|$.

Therefore for any given suspension semiflow over such quadratic maps $f_{a}$ with roof function having logarithmic growth near 0 we can apply Theorem $\mathrm{A}$, and obtain a large deviation bound for these special flows.

\subsection{Lorenz and geometric Lorenz attractors}

The $C^{1+\alpha}$ map $f:[-1,1] \backslash\{0\} \rightarrow[-1,1]$ obtained as the quotient map of the Poincare first return map $R$ presented in Section 1.3 through projection along the leaves of the stable foliation satisfies the following conditions, which define a Lorenz-like map:

1. there are constants $c>0$ and $\sigma>1$ such that for every $n \geq 1$ and for all $x \in$ $[-1,1] \backslash \cup_{0 \leq j<n} f^{-n}\{0\}$ we have $\left|\left(f^{n}\right)^{\prime}(x)\right| \geq c \sigma^{n}$;

2. $f$ has a dense orbit;

3. $f\left(0^{+}\right)=-1, f\left(0^{-}\right)=1, f(1) \in(0,1)$ and $f(-1) \in(-1,0)$.

Note in particular that there are no critical points and that for some $k \geq 1$ the map $g=f^{k}$ satisfies the conditions of Section 2.2. (If $\sigma>\sqrt{2}$ then $f$ is even locally eventually onto, see e.g. [44], thus transitive.) For exponentially slow recurrence to the singularities see Section 6, So we can obtain a large deviation bound for $g$ which easily gives a large deviation bound for $f$.

Indeed, assume without loss of generality that $\mu(\varphi)=0$ and that for all small $\varepsilon>0$ we have $\operatorname{Leb}\left\{S_{n}^{g} \varphi>n \varepsilon\right\}<C e^{-\zeta n}$ for some $C(\varepsilon), \zeta(\varepsilon)>0$ and every $n>0$. It is enough to argue for a bounded and continuous $\varphi$ as explained in Section 3. Then for $m>0$ we can write $m=n k+p$ with $n>0$ and $0 \leq p<k-1$ and also

$$
\begin{aligned}
\frac{1}{m} S_{m}^{f} \varphi & =\frac{1}{n k+p}\left(S_{p}^{f}\left(\varphi \circ f^{n k}\right)+S_{n k}^{f} \varphi\right)=\frac{1}{n k+p}\left(S_{p}^{f}\left(\varphi \circ f^{n k}\right)+\sum_{i=0}^{n-1} S_{n}^{g}\left(\varphi \circ f^{i}\right)\right) \\
& \leq \frac{p \sup |\varphi|}{n k+p}+\frac{1}{k+p / n} \sum_{i=0}^{n-1} \frac{1}{n} S_{n}^{g}\left(\varphi \circ f^{i}\right) \leq \frac{p}{m} \sup |\varphi|+\frac{1}{k} \sum_{i=0}^{n-1} \frac{1}{n} S_{n}^{g}\left(\varphi \circ f^{i}\right) .
\end{aligned}
$$

Given $\varepsilon>0$ take $m$ so big that $p \sup |\varphi| / m<\varepsilon / 2$, note that $\mu\left(\varphi \circ f^{i}\right)=0$ for all $i \geq 0$ and

$$
\left\{\frac{1}{m} S_{m}^{f} \varphi>\varepsilon\right\} \subseteq \bigcup_{i=0}^{n-1}\left\{\frac{1}{n} S_{n}^{g}\left(\varphi \circ f^{i}\right)>\frac{\varepsilon}{2 k}\right\} .
$$


This shows how to reduce the problem of large deviations for bounded observables to the same problem for a finite power of the transformation.

To deduce Corollary $\mathrm{C}$, since the reduction to a large deviation bound for the map $f$ is the content of Section 4, all we need to do here is to explain how we deduce a large deviation bound for $R$ from a similar bound for the map $f$. For this we strongly use the uniform contraction along the leaves of the stable foliation on the global cross-section $S$ to obtain the following relation. Denote by $P: S \rightarrow[-1,1]$ the projection $(x, y, 1) \mapsto x$.

Lemma 2.1. Let $\varepsilon>0$ and a bounded continuous function $\psi: U \rightarrow \mathbb{R}$ be given in a neighborhood $U$ of the geometric Lorenz attractor $\Lambda$. Define $\varphi: S \backslash \ell \rightarrow \mathbb{R}$ by $\varphi(x, y, 1)=$ $\int_{0}^{\tau(x, y, 1)} \psi\left(X^{t}(x, y, 1)\right) d t$, where $\tau(x, y, 1)$ is the first return time to $S$ of the point $(x, y, 1) \in S$. Assume without loss of generality that $\mu(\varphi)=0$ where $\mu$ is a R-invariant probability measure such that $\tau$ is $\mu$-integrable.

Then there exist integers $N, k>1, a$ small $\delta>0$, a constant $\gamma>0$ dependent on $\psi$ and the flow only, and a continuous function $l:[-1,1] \backslash \cup_{i=0}^{k-1} f^{-i}\{0\} \rightarrow \mathbb{R}$ with logarithmic growth near the set $S_{k}=\cup_{i=0}^{k-1} f^{-i}\{0\}$ such that for all $n>N$

$$
\left\{\left|\frac{1}{n} S_{n}^{R^{k}} \varphi\right|>3 \varepsilon\right\} \subseteq P^{-1}\left(\left\{\frac{1}{n} S_{n}^{f^{k}} \Delta_{\delta}>\frac{\varepsilon}{\gamma}\right\} \cup\left\{\left|\frac{1}{n} S_{n}^{f^{k}} l\right|>\varepsilon\right\}\right) .
$$

This reduces the problem of estimating the Lebesgue measure of the left hand side set in (2.1) to the estimation of the measure of the right hand side set, transferring the problem to the dynamics of $g=f^{k}$, which is the subject of Section 2.2] and Section 6 .

Proof. According to the construction of geometric Lorenz flows, there are $C>0$ and $0<\lambda<1$ such that given $x \in[-1,1] \backslash\{0\}$ and two distinct values $y_{1}, y_{2} \in[-1,1]$

$$
\operatorname{dist}\left(R^{k}\left(x, y_{1}, 1\right), R^{k}\left(x, y_{2}, 1\right)\right) \leq C \lambda^{k} \text { for all } 1 \leq k \leq n,
$$

where $n \geq 1$ is the first time the orbit of the points hit the singular line, corresponding to the stable foliation of the singularity of the flow. These hitting times depend only on the orbit of $x$ under the map $f$ and correspond to times $n$ for which $f^{n}(x)=0$. But $X_{0}=\cup_{n \geq 0} f^{-n}(\{0\})$ is denumerable. Thus the corresponding set of points in $S$, given by the lines $\{x\} \times[-1,1] \times\{1\}$ for $x \in X_{0}$, has zero area on $S$. Therefore for a full Lebesgue measure subset of $S$ we have (2.2) for all $k \geq 1$.

Moreover since $\left(x, y_{1}, 1\right),\left(x, y_{2}, 1\right)$ belong to the same stable manifold, then for all times $t>0$ we have

$$
\operatorname{dist}\left(X^{t}\left(x, y_{1}, 1\right), X^{t}\left(x, y_{2}, 1\right)\right) \leq \kappa \cdot\left|y_{1}-y_{2}\right|,
$$

for a constant $\kappa>0$ depending only on the angles between the surface $S$ and the stable leaves of the flow through points of $S$ (which is uniformly bounded by the compactness of $S$ ). Note that $\varphi$ is continuous on $S \backslash \ell$ and

$$
|\varphi(x, y, 1)| \leq \tau(x, y, 1) \cdot \sup |\psi| \leq-C_{0} \cdot \log |x| \cdot \sup |\psi|
$$


for a constant $C_{0}>0$, since $\tau$ grows near $\ell$ like the logarithm of the distance to $\ell$. Then it is clear that for $y, w \in[-1,1]$ and $n>1$

$$
\mid \frac{1}{n} \sum_{j=0}^{n-1}\left(\varphi\left(R^{j}(x, y, 1)\right)-\varphi\left(R^{j}(x, w, 1)\right) \mid \leq \frac{1}{n} \sum_{j=0}^{n-1} \bar{\varphi}_{j}(x)\right.
$$

where $\left(x_{j}, y_{j}, 1\right)=R^{j}(x, y, 1)$ for $j \geq 0,(x, y, 1) \in S$, and

$$
\bar{\varphi}_{j}(x)=\sup _{y, w \in[-1,1]}\left|\varphi\left(R^{j}(x, y, 1)\right)-\varphi\left(R^{j}(x, w, 1)\right)\right| .
$$

Let $\varepsilon>0$ be given. Choose a small $\delta>0$ and $\eta>0$ such that $-C_{0} \kappa \eta \log \delta<\varepsilon / 3$ and $\kappa \eta \leq \sup |\psi|$. Let $\xi>0$ satisfy

$$
\operatorname{dist}\left((x, y, z),\left(x^{\prime}, y^{\prime}, z^{\prime}\right)\right)<\xi \Longrightarrow\left|\psi(x, y, z)-\psi\left(x^{\prime}, y^{\prime}, z^{\prime}\right)\right|<\eta .
$$

Then we may find by (2.2) a $j_{0}=j_{0}(\eta) \geq 1$ such that $\left|y_{j}-w_{j}\right| \leq \xi / \kappa$ for $j>j_{0}$ and any pair $y, w$ in the same vertical line. Thus we also get after (2.3), (2.5) and the choices of $\varepsilon, \delta$ and $\eta$

$$
\begin{aligned}
\bar{\varphi}_{j}(x) & \leq-C_{0} \log \left|x_{j}\right| \cdot \sup _{0<t<-C_{0} \log \left|x_{j}\right|}\left|\psi\left(X^{t}\left(x_{j}, y_{j}, 1\right)\right)-\psi\left(X^{t}\left(x_{j}, w_{j}, 1\right)\right)\right| \\
& \leq-C_{0} \log \left|x_{j}\right| \cdot \kappa \eta \leq C_{0} \kappa \eta \cdot \Delta_{\delta}\left(x_{j}\right)+\varepsilon / 2 .
\end{aligned}
$$

Take a continuous $l:[-1,1] \backslash\{0\} \rightarrow \mathbb{R}$ such that for some $0<a<\varepsilon / 3$

1. $\min _{y \in[-1,1]} \varphi\left(R^{j_{0}}(x, y, 1)\right)-a \leq l(x) \leq a+\max _{y \in[-1,1]} \varphi\left(R^{j_{0}}(x, y, 1)\right)$; and

2. $\mu(l \circ P)=\mu(\varphi)$.

Note that $\varphi$ is $\mu$-integrable: this follows from the boundedness assumption on $\psi$ and by the $\mu$-integrability of $\tau$ after (2.4). Observe that $l$ as above has logarithmic growth near $\mathcal{S}_{k}$ by definition.

To obtain such function $l$ disintegrate $\mu$ along the measurable partition of $S$ given by the vertical lines $\{x\} \times[-1,1] \times\{1\}$ and define $l_{0}(x)=\int \varphi d \mu_{x}$. Then approximate $l_{0}$ by a continuous function $l_{1}$ such that $\int\left|l_{0}-l_{1}\right| \circ P d \mu<\varepsilon / 3$ (through e.g. a convolution). Now for some $-\varepsilon / 3<a<\varepsilon / 3$ the function $l=l_{1}+a$ satisfies conditions $1-2$ above.

Now for $n>0$ using (2.6), $f \circ P=P \circ R$ and summing over orbits of $R^{k}$ and $f^{k}$

$$
\begin{aligned}
\left|S_{n}(l \circ P)-S_{n} \varphi\right|(x, y, 1) & \leq|l \circ P-\varphi|(x, y, 1)+\left|S_{n-1}(l \circ P-\varphi)\right|(x, y, 1) \\
& \leq 2 \sup |\psi| C_{0} \log |x|+a+\sum_{j=1}^{n-1}\left(C_{0} \kappa \eta \Delta_{\delta}\left(f^{j k}(x)\right)+\frac{\varepsilon}{3}+a\right) \\
& \leq 2 \sup |\psi| C_{0}\left(\log \delta^{-1}+\Delta_{\delta}(x)\right)+\frac{2 n \varepsilon}{3}+C_{0} \kappa \eta \cdot S_{n-1} \Delta_{\delta}\left(f^{k}(x)\right) \\
& \leq 2 \sup |\psi| C_{0} \log \delta^{-1}+\frac{2 n \varepsilon}{3}+C_{0}(\kappa \eta+2 \sup |\psi|) \cdot S_{n} \Delta_{\delta}(x) .
\end{aligned}
$$


Observe that

$$
\left\{\left|\frac{1}{n} S_{n}^{R^{k}} \varphi\right|>3 \varepsilon\right\} \subseteq\left\{\left|\frac{1}{n}\left(S_{n}^{R^{k}}(l \circ P)-S_{n}^{R^{k}} \varphi\right)\right|>2 \varepsilon\right\} \cup\left\{\frac{1}{n}\left|S_{n}^{R^{k}}(l \circ P)\right|>\varepsilon\right\} .
$$

From (2.7), setting $\gamma_{1}=2 \sup |\psi| C_{0} \log \delta^{-1}$ and $\gamma_{2}=C_{0}(\kappa \eta+2 \sup |\psi|)$ we obtain for $n$ big enough

$$
\frac{1}{n}\left(S_{n}^{R^{k}}(l \circ P)-S_{n}^{R^{k}} \varphi\right) \leq \frac{2 \varepsilon}{3}+\frac{\gamma_{1}}{n}+\frac{\gamma_{2}}{n} \cdot S_{n}^{f^{k}} \Delta_{\delta} \circ P \leq \varepsilon+\frac{\gamma_{2}}{n} \cdot S_{n}^{f^{k}} \Delta_{\delta} \circ P
$$

where $\gamma_{2} \leq 3 C_{0}$ sup $|\psi|$ by the choice of $\eta$. Hence

$$
\left\{\left|\frac{1}{n}\left(S_{n}^{R^{k}}(l \circ P)-S_{n}^{R^{k}} \varphi\right)\right|>2 \varepsilon\right\} \subseteq P^{-1}\left\{\frac{1}{n} S_{n}^{f^{k}} \Delta_{\delta}>\frac{\varepsilon}{3 C_{0} \sup |\psi|}\right\}
$$

and this together with (2.8) completes the proof of the lemma.

\section{Large deviations for observables with logarithmic growth near singularities}

The main bound on large deviations for suspension semiflows over a non-uniformly expanding base will be obtained from the following large deviation statement for nonuniformly expanding transformations.

Theorem 3.1. Let $f: M \rightarrow M$ be a regular $C^{1+\alpha}$ local diffeomorphism on $M \backslash \mathcal{S}$ where $\mathcal{S}$ is a non-flat critical set and $\alpha \in(0,1)$. Assume that $f$ is a non-uniformly expanding map with exponentially slow recurrence to the singular set $\mathcal{S}$ and let $\varphi: M \backslash \mathcal{S} \rightarrow \mathbb{R}$ be a continuous map which has logarithmic growth near $\mathcal{S}$. Moreover assume that there exists a unique equilibrium state $\mu$ with respect to $\log J$ which is absolutely continuous. Then for any given $\omega>0$

$$
\limsup _{n \rightarrow+\infty} \frac{1}{n} \log \operatorname{Leb}\left\{x \in M:\left|\frac{1}{n} S_{n} \varphi(x)-\mu(\varphi)\right| \geq \omega\right\}<0 .
$$

Proof. Define

$$
\varphi_{k}=\xi_{k} \circ \varphi \text { where } \xi_{k}(x)=\left\{\begin{array}{ll}
k & \text { if } x \geq k \\
x & \text { if }|x|<k \\
-k & \text { if } x \leq-k
\end{array}, k \geq 1 .\right.
$$

Then $\varphi_{k}: M \rightarrow \mathbb{R}$ is continuous for all $k \geq 1, \varphi_{k}(x) \underset{k \rightarrow \infty}{\longrightarrow} \varphi(x)$ for all $x \in M \backslash \mathcal{S}$ and $\left|\varphi-\varphi_{k}\right| \leq|\varphi| \chi_{\{|\varphi|>k\}}$. Moreover we clearly have for all $n, k \geq 1$

$$
S_{n} \varphi_{k}-S_{n}\left|\varphi-\varphi_{k}\right| \leq S_{n} \varphi=S_{n} \varphi_{k}+S_{n}\left(\varphi-\varphi_{k}\right) \leq S_{n} \varphi_{k}+S_{n}\left|\varphi-\varphi_{k}\right| .
$$


Observe that, since $\varphi$ has logarithmic growth near $\mathcal{S}$ (see (1.4)), for any given $c, \varepsilon_{0}>0$ we may choose $\varepsilon_{1}, \delta_{1}>0$ such that the exponential slow recurrence condition (1.2) is true and $K \cdot \varepsilon_{1} \leq \varepsilon_{0}$. Then choose $k \geq 1$ very big so that $\{|\varphi|>k\} \subseteq B\left(\mathcal{S}, \delta_{1}\right)$. From (3.1) we obtain the following inclusions

$$
\begin{aligned}
\left\{\frac{1}{n} S_{n} \varphi>c\right\} & \subseteq\left\{\frac{1}{n} S_{n} \varphi_{k}+\frac{1}{n} S_{n}\left|\varphi-\varphi_{k}\right|>c\right\} \subseteq\left\{\frac{1}{n} S_{n} \varphi_{k}>c-K \varepsilon_{1}\right\} \cup\left\{\frac{1}{n} S_{n}\left|\varphi-\varphi_{k}\right|>K \varepsilon_{1}\right\} \\
& \subseteq\left\{\frac{1}{n} S_{n} \varphi_{k}>c-\varepsilon_{0}\right\} \cup\left\{\frac{1}{n} S_{n} \Delta_{\delta_{1}} \geq \varepsilon_{1}\right\}
\end{aligned}
$$

where in (3.2) we use the assumption that $\varphi$ is of logarithmic growth near $\mathcal{S}$ and the choices of $\varepsilon_{1}, \delta_{1}$. Analogously we get with opposite inequalities

$$
\begin{aligned}
\left\{\frac{1}{n} S_{n} \varphi<c\right\} & \subseteq\left\{\frac{1}{n} S_{n} \varphi_{k}-\frac{1}{n} S_{n}\left|\varphi-\varphi_{k}\right|<c\right\} \subseteq\left\{\frac{1}{n} S_{n} \varphi_{k}<c+K \varepsilon_{1}\right\} \cup\left\{\frac{1}{n} S_{n}\left|\varphi-\varphi_{k}\right|>K \varepsilon_{1}\right\} \\
& \subseteq\left\{\frac{1}{n} S_{n} \varphi_{k}<c+\varepsilon_{0}\right\} \cup\left\{\frac{1}{n} S_{n} \Delta_{\delta_{1}} \geq \varepsilon_{1}\right\}
\end{aligned}
$$

From (3.2) and (3.3) we see that to get the bound for large deviations in the statement of Theorem 3.1 it suffices to obtain a large deviation bound for the continuous function $\varphi_{k}$ with respect to the same transformation $f$ and to have exponentially slow recurrence to the singular set $\mathcal{S}$.

To obtain this large deviation bound, we use the following result already obtained for continuous observables over non-uniformly expanding transformations in our setting, see [7].

Theorem 3.2. Let $f: M \rightarrow M$ be a local diffeomorphism outside a non-flat singular set $\mathcal{S}$ which is non-uniformly expanding and has slow recurrence to $\mathcal{S}$. For $\omega_{0}>0$ and a continuous function $\varphi_{0}: M \rightarrow \mathbb{R}$ there exists $\varepsilon, \delta>0$ arbitrarily close to 0 such that, writing

$$
A_{n}=\left\{x \in M: \frac{1}{n} S_{n} \Delta_{\delta}(x) \leq \varepsilon\right\} \quad \text { and } \quad B_{n}=\left\{x \in M: \inf \left\{\left|\frac{1}{n} S_{n} \varphi_{0}(x)-\eta\left(\varphi_{0}\right)\right|: \eta \in \mathbb{E}\right\}>\omega_{0}\right\}
$$

we get limsup $\sup _{n \rightarrow+\infty} \frac{1}{n} \log \operatorname{Leb}\left(A_{n} \cap B_{n}\right)<0$.

Recall that $\mathbb{E}$ is the set of all equilibrium states of $f$ with respect to the potential $\log J$.

Note that exponentially slow recurrence implies $\lim \sup _{n \rightarrow+\infty} \frac{1}{n} \operatorname{Leb}\left(M \backslash A_{n}\right)<0$. Under this assumption Theorem 3.2 ensures that for $(\varepsilon, \delta)$ close enough to $(0,0)$ we get $\limsup _{n \rightarrow+\infty} \frac{1}{n} \log \operatorname{Leb}\left(B_{n}\right)<0$. To use this we also need that $\mathbb{E}$ consists only of the unique absolutely continuous invariant probability measure $\mu$. Under this uniqueness assumption we have $\mathbb{E}=\{\mu\}$ in Theorem 3.2 and take $\omega, \varepsilon_{0}>0$ small, choose $k \geq 1$ as before, set $\varphi_{0}=\varphi_{k}$ and $\omega_{0}=\omega+\varepsilon_{0}$. In (3.2) set $c=\mu\left(\varphi_{0}\right)-\omega$ and in (3.3) set $c=\mu\left(\varphi_{0}\right)+\omega$. Then we have the inclusion

$$
\left\{\left|\frac{1}{n} S_{n} \varphi-\mu(\varphi)\right|>\omega\right\} \subseteq\left\{\left|\frac{1}{n} S_{n} \varphi_{0}-\mu\left(\varphi_{0}\right)\right|>\omega_{0}\right\} \cup\left\{\frac{1}{n} S_{n} \Delta_{\delta_{1}} \geq \varepsilon_{1}\right\} .
$$


By Theorem 3.2 we may find $\varepsilon, \delta>0$ small enough so that the exponentially slow recurrence holds also for the pair $(\varepsilon, \delta)$ and hence

$$
\limsup _{n \rightarrow+\infty} \frac{1}{n} \log \operatorname{Leb}\left\{\left|\frac{1}{n} S_{n} \varphi_{0}-\mu\left(\varphi_{0}\right)\right|>\omega_{0}\right\}<0 .
$$

Finally the choice of $\varepsilon_{1}, \delta_{1}$ according to the condition on exponential slow recurrence to $\mathcal{S}$ ensures that the Lebesgue measure of the right hand subset in (3.4) is also exponentially small when $n \rightarrow \infty$. This together with (3.5) concludes the proof of Theorem 3.1.

\section{Large deviations and the dynamics on the base}

Here we show how the large deviation bound for a semiflow over a non-uniformly expanding base can be deduced from a large deviation bound for the base dynamics, under a logarithmic growth condition on the roof function.

\subsection{Reduction to the base dynamics}

Let $\psi: M_{r} \rightarrow \mathbb{R}$ be continuous and bounded. For $T>0$ and $z=(x, s)$ with $x \in M$ and $0 \leq s<r(x)<\infty$ we can write

$$
\int_{0}^{T} \psi\left(X^{t}(z)\right) d t=\int_{s}^{r(x)} \psi\left(X^{t}(x, 0)\right) d t+\sum_{j=1}^{n-1} \int_{0}^{r\left(f^{j}(x)\right)} \psi\left(X^{t}\left(f^{j}(x), 0\right)\right) d t+\int_{0}^{T+s-S_{n} r(x)} \psi\left(X^{t}\left(f^{n}(x), 0\right)\right) d t,
$$

where $n=n(x, s, T) \in \mathbb{N}$ is the "lap number" such that $S_{n} r(x) \leq s+T<S_{n+1} r(x)$.

Setting $\varphi(x)=\int_{0}^{r(x)} \psi(x, 0) d t$ we obtain

$$
\frac{1}{T} \int_{0}^{T} \psi\left(X^{t}(z)\right) d t=\frac{1}{T} S_{n} \varphi(x)-\frac{1}{T} \int_{0}^{s} \psi\left(X^{t}(x, 0)\right) d t+\frac{1}{T} \int_{0}^{T+s-S_{n} r(x)} \psi\left(X^{t}\left(f^{n}(x), 0\right)\right) d t .
$$

Clearly we can bound the sum $I=I(x, s, T)$ of the two integral terms on the right hand side above by

$$
I=I(x, s, T) \leq\left(2 \frac{s}{T}+\frac{S_{n+1} r(x)-S_{n} r(x)}{T}\right) \cdot\|\psi\|,
$$

where $\|\psi\|=\sup |\psi|$. Observe that for a given $\omega>0$ and for $0 \leq s<r(x)$ and $n=n(x, s, T)$

$$
\left\{(x, s) \in M_{r}:\left|\frac{1}{T} S_{n} \varphi(x)+I(x, s, T)-\frac{\mu(\varphi)}{\mu(r)}\right|>\omega\right\}
$$

is contained in

$$
\left\{(x, s) \in M_{r}:\left|\frac{1}{T} S_{n} \varphi(x)-\frac{\mu(\varphi)}{\mu(r)}\right|>\frac{\omega}{2}\right\} \cup\left\{(x, s) \in M_{r}: I(x, s, T)>\frac{\omega}{2}\right\} .
$$

Note that if $\psi \equiv 0$ then we need only consider the left hand subset of (4.3) in what follows. Now we bound the $\lambda$-measure of each subset above assuming that $\psi$ is not identically zero. 


\subsection{Using the roof function as an observable over the base dynamics}

We start with the right hand subset in (4.3). Take $N \geq 1$ big enough so that $N\|\psi\|>2$ and note that for any given $T, \omega>0$ using (4.1) and $n=n(x, s, T)$

$$
\begin{aligned}
& \lambda\left\{I>\frac{\omega}{2}\right\}=\int d \operatorname{Leb}(x) \int_{0}^{r(x)} d s\left(\chi_{(\omega / 2,+\infty)} \circ I\right)(x, s, T) \\
& \leq \operatorname{Leb}\left\{r>\frac{\omega T}{2 N\|\psi\|}\right\}+\frac{\omega T}{2 N\|\psi\|} \sum_{i=0}^{\left.T T / r_{0}\right]+1} \operatorname{Leb}\left\{\frac{\left|S_{i+1} r-S_{i} r\right|}{T}>\frac{N\|\psi\|-2}{2 N\|\psi\|} \omega\right\},
\end{aligned}
$$

where in the right hand summand we restrict to points $x \in M$ such that $2 N\|\psi\| r(x) \leq \omega T$ and $S_{i} r(x) \leq T<S_{i+1} r(x)$ for each possible lap number $i \in \mathbb{N}$. Note that since $r$ is bounded from below $r \geq r_{0}>0$ we have $T \geq r_{0} n$ which gives an upper bound $\left[T / r_{0}\right]+1$ for the possible lap numbers appearing in the summation above, where $[t]$ denotes $\max \{k \in \mathbb{Z}: k \leq t\}$, the integer part of $t \geq 0$. In (4.4) we have also used the relations

$$
\frac{2 s}{T}<\frac{2 r}{T} \leq \frac{\omega}{N\|\psi\|} \quad \text { and } \quad \frac{\omega}{2}-\frac{\omega}{N\|\psi\|}=\frac{N\|\psi\|-2}{2 N\|\psi\|} \cdot \omega .
$$

On the one hand, since $r$ grows as the logarithm of the distance to $\mathcal{S}$, we have that the left hand summand in (4.4) is bounded by

$$
\operatorname{Leb}\left\{x \in M: \operatorname{dist}(x, \mathcal{S}) \leq \exp \left(-C \cdot \frac{\omega T}{2 N\|\psi\|}\right)\right\} \leq e^{-C \cdot \kappa \cdot \omega T \| /(2 N\|\psi\|)},
$$

where $C>0$ is a constant depending on $r$ only, and we use condition (S4) on the geometry of $\mathcal{S}$. On the other hand, from $T \geq S_{i} r(x) \geq r_{0} i$ we get the following upper bound for the summands in the right hand side of (4.4) for each $i=0, \ldots,\left[T / r_{0}\right]+1$

$$
\begin{aligned}
& \text { Leb }\left\{\frac{\left|S_{i+1} r-S_{i} r\right|}{i}>\left(\frac{N\|\psi\|-2}{2 N\|\psi\|} r_{0}\right) \cdot \omega\right\} \quad\left(\text { let } r_{0}^{\prime}=\frac{N\|\psi\|-2}{2 N\|\psi\|} r_{0}\right) \\
& \leq \text { Leb }\left\{\left|\frac{1}{i} S_{i} r-\mu(r)\right|>\frac{\omega r_{0}^{\prime}}{2}\right\}+\operatorname{Leb}\left\{\left|\frac{1}{i} S_{i+1} r-\mu(r)\right|>\frac{\omega r_{0}^{\prime}}{2}\right\} \leq 2 C_{0} e^{-\beta i}
\end{aligned}
$$

for some constants $C_{0}, \beta>0$, since we have a large deviation bound for the observable $r$ with respect to the unique physical measure $\mu$ for $f$. Recall (see Section 3 ) that we took $r$ to be $\mu$-integrable, continuous on $M \backslash \mathcal{S}$ and with logarithmic growth near $\mathcal{S}$, and $f$ is a non-uniformly expanding map with exponentially slow recurrence to $\mathcal{S}$. Consequently we can bound the summation in (4.4) as

$$
\frac{\omega T}{2 N\|\psi\|} \cdot 2 C_{0} \sum_{i=0}^{\left[T / r_{0}\right]+1} e^{-\beta i} \leq \frac{C \omega T}{2 N\|\psi\|} \cdot e^{-\beta T / r_{0}}
$$


for a constant $C>0$ depending on $f, r, \omega$ and $\psi$. Altogether we see that $\lambda\{I>\omega / 2\}$ is bounded by twice the maximum of the summands in (4.4).

From this we obtain

$$
\limsup _{T \rightarrow \infty} \frac{1}{T} \log \lambda\left\{I>\frac{\omega}{2}\right\}<0,
$$

as long as we take $\omega>0$ small enough.

\subsection{Using $\varphi$ as an observable over the base dynamics}

Now for the left hand subset in (4.3), note first that for $\mu$-and Leb-almost every $x \in M$ and every $0 \leq s<r(x)$

$$
\frac{S_{n} r(x)}{n} \leq \frac{T+s}{n} \leq \frac{S_{n+1} r(x)}{n} \quad \text { so } \quad \frac{n(x, s, T)}{T} \underset{T \rightarrow \infty}{\longrightarrow} \frac{1}{\mu(r)}
$$

We also have (recall that $n=n(x, s, T)$ )

$$
\left|\frac{1}{T} S_{n} \varphi-\frac{\mu(\varphi)}{\mu(r)}\right| \leq\left|\frac{n}{T} \cdot \frac{S_{n} \varphi}{n}-\frac{n}{T} \mu(\varphi)\right|+\left|\frac{n}{T} \mu(\varphi)-\frac{\mu(\varphi)}{\mu(r)}\right| \leq \frac{n}{T}\left|\frac{S_{n} \varphi}{n}-\mu(\varphi)\right|+|\mu(\varphi)|\left|\frac{n}{T}-\frac{1}{\mu(r)}\right| .
$$

Hence the left hand subset in (4.3) is contained in

$$
\left\{(x, s) \in M_{r}: \frac{n}{T}\left|\frac{S_{n} \varphi}{n}-\mu(\varphi)\right|>\frac{\omega}{4}\right\} \cup\left\{(x, s) \in M_{r}:\left|\frac{n}{T}-\frac{1}{\mu(r)}\right|>\frac{\omega}{4|\mu(\varphi)|}\right\} .
$$

Notice that the $\lambda$-measure of the right hand subset of (4.10) is bounded from above by

$$
\begin{aligned}
& \lambda\left\{\left|\frac{n}{T}-\frac{1}{\mu(r)}\right|>\frac{\omega}{4|\mu(\varphi)|} \& r \leq T\right\}+\lambda\{r>T\} \\
& \leq T \sum_{i=0}^{\left[T / r_{0}\right]+1} \sum_{j=0,1} \operatorname{Leb}\left\{x \in M:\left|\frac{i}{S_{i+j} r}-\frac{1}{\mu(r)}\right|>\frac{\omega}{|\mu(\varphi)|}\right\}+\int_{\{r>T\}}^{r d \text { Leb }}
\end{aligned}
$$

where we have used the relation (4.9), for small enough $\omega>0$ and big enough $T$ and

$n$. The first summand in (4.11) can be bounded using the large deviation bound for the observable $r$ as before: there are constants $C_{0}, \beta>0$ such that

$$
\operatorname{Leb}\left\{x \in M:\left|\frac{i}{S_{i+j} r}-\frac{1}{\mu(r)}\right|>\frac{\omega}{|\mu(\varphi)|}\right\} \leq C_{0} e^{-\beta i} \quad \text { for } \quad j=0,1,
$$

and so for some constant $C_{1}>0$ depending only on $f, r$ and $\omega$ we get

$$
T \sum_{i=0}^{\left[T / r_{0}\right]+1} \sum_{j=0,1} \operatorname{Leb}\left\{x \in M:\left|\frac{i}{S_{i+j} r}-\frac{1}{\mu(r)}\right|>\frac{\omega}{|\mu(\varphi)|}\right\} \leq C_{1} T e^{-\beta T / r_{0}}
$$


The second summand in (4.11) is easily bounded using condition (S4) as follows: for big enough $T>0$ such that $i>[T]$ implies $(i+1) e^{c_{0} i}<1$, where $c_{0}=-\kappa \log \rho /(2 K)>0$, we have as in (4.5)

$$
\begin{aligned}
\int_{\{r>T\}}^{r d \operatorname{Leb}} & \leq \sum_{i \geq[T]} \int_{i}^{i+1} r d \text { Leb } \leq \sum_{i \geq[T]}(i+1) \operatorname{Leb}\{r>i\} \\
& \leq C_{\kappa} \sum_{i \geq[T]}(i+1) e^{-2 c_{0} i} \leq C_{\kappa} \sum_{i \geq[T]} e^{-c_{0} i} \leq C_{2} \cdot e^{-c_{0} T}
\end{aligned}
$$

for a positive constant $C_{2}>0$ depending only on $f$.

Finally the left hand subset of (4.10) is contained in the following union

$$
\left\{(x, s) \in M_{r}:\left|\frac{T}{n}-\mu(r)\right|>\frac{\mu(r)}{2} \cdot \omega\right\} \cup\left\{(x, s) \in M_{r}:\left|\frac{S_{n} \varphi}{n}-\mu(\varphi)\right|>\frac{\mu(r)}{2} \cdot \frac{\omega}{4}\right\} .
$$

Again for small $\omega>0$ the $\lambda$-measure of the left hand subset in (4.13) is exponentially small with $T$, using similar arguments to (4.11) and (4.12). For the right hand subset in (4.13) we use the large deviation bound for the observable $\varphi$ with respect to $f$, since $\varphi$ has also logarithmic growth near $\mathcal{S}$. In fact $|\varphi(x)| \leq \int_{0}^{r(x)}|\psi(x, s)| d t \leq r(x) \cdot\|\psi\|$ for $x \in M \backslash \mathcal{S}$ because $\psi: M_{r} \rightarrow \mathbb{R}$ is bounded. We can estimate the $\lambda$-measure of the right hand subset in (4.13) as in (4.11) through (4.12) (or as in (4.6) and (4.7)), obtaining constants $C_{3}, \gamma>0$ depending on $f, r$ and $\omega$ such that

$$
\lambda\left\{\left|\frac{S_{n} \varphi}{n}-\mu(\varphi)\right|>\frac{\mu(r)}{2} \cdot \frac{\omega}{4}\right\} \leq C_{3} T e^{-\gamma n}
$$

From this we conclude

$$
\underset{T \rightarrow \infty}{\limsup } \frac{1}{T} \log \lambda\left\{\left|\frac{1}{T} S_{n} \varphi-\frac{\mu(\varphi)}{\mu(r)}\right|>\frac{\omega}{2}\right\}<0 .
$$

Putting (4.8) and (4.14) together, as long as we have a result on large deviations for continuous observables in $M \backslash \mathcal{S}$ with logarithmic growth near $\mathcal{S}$, with respect to the dynamics of $f$ and the Lebesgue measure, and the volume of neighborhoods of $\mathcal{S}$ is comparable to a power of the radius, we are able to prove the Main Theorem for the suspension flow over $f$.

We have obtained the large deviation bounds needed for the base dynamics in Section 3, so the proof of Theorem A is complete.

\section{The Entropy Formula for non-uniformly expanding maps}

Here we obtain the Entropy Formula when $f$ is a non-uniformly expanding map with slow recurrence to the singular set. The singular set $\mathcal{S}$ is formed by critical points of $f$ and points where $f$ is either not defined, is not continuous or is not differentiable. Recall from the Introduction that $\psi=\log \left\|(D f)^{-1}\right\|$ and that $J=|\operatorname{det} D f|$. 
Theorem 5.1. Let $f: M \rightarrow M$ be a non-uniformly expanding map with slow recurrence to the non-flat singular set $\mathcal{S}$. Let $\mu \in \mathcal{M}_{f}$ be such that $\mu$ is $f$-ergodic, $h_{\mu}(f)=\mu(\log J),-\infty<\mu(\psi)<0$ and for every given $\varepsilon>0$ there exists $\delta>0$ so that $\mu\left(\Delta_{\delta}\right)<\varepsilon$. Then $\mu \ll \mathrm{Leb}$ and consequently $\mu \in \overline{\mathrm{CO}}(\mathbb{F})$.

Reciprocally, let $\mu \in \mathcal{M}_{f}$ be such that $\mu$ is absolutely continuous with respect to Leb and assume that $\Delta_{\delta}$ is $\mu$-integrable. Then $h_{\mu}(f)=\mu(\log J)$.

Here $\overline{c o}(\mathbb{F})$ is the weak* closure of the convex hull of the finite set $\mathbb{F}$ of ergodic physical probability measures for $f$. Clearly this is a particular case of the more general Entropy Formula obtained by Ledrappier and Young [40, 41] applied to maps with singularities and/or criticalities. For $C^{2}$ endomorphisms (i.e. smooth maps with criticalities but no singularities) see Bahnmüller and Liu [42, 14] for a general statement. A similar result for piecewise smooth one-dimensional maps with finitely many branches was obtained by Ledrappier [39].

As an easy corollary we deduce that $\overline{\mathrm{co}}(\mathbb{F})$ is isolated among the set $\mathbb{E}$ of all equilibrium states of $f$ with respect to $J=\log |\operatorname{det} D f|$, which might be of independent interest for the ergodic theory of non-uniformly expanding transformations.

Corollary 5.2. Let $f: M \rightarrow M$ be a non-uniformly expanding map with slow recurrence to the non-flat singular set $\mathcal{S}$. Then there exists a weak neighborhood $\mathcal{U}$ of $\overline{\mathrm{co}}(\mathbb{F})$ in $\mathcal{M}_{f}$ such that $\mathcal{U} \cap \mathbb{E}=\overline{\mathrm{CO}}(\mathbb{F})$.

Proof. Take any weak ${ }^{*}$ neighborhood $\mathcal{U}$ of $\overline{\mathrm{co}}(\mathbb{F})$ such that every $\mu \in \mathcal{U}$ satisfies $\mu(\psi)<0$. Hence every $\mu \in U \cap \mathbb{E}$ satisfies the conditions of Theorem 5.1, thus $\mu \in \overline{\mathrm{co}}(\mathbb{F})$.

Note that whenever the Entropy Formula and its reciprocal hold for measures close to $\mathbb{F}$ then the argument proving Corollary $[5.2$ is applicable and we deduce that $\mathbb{F}$ is isolated in $\mathbb{E}$. The proof of Theorem 5.1 is longer and occupies the rest of this section.

\subsection{Hyperbolic times}

Here we present some technical results for the study of non-uniformly expanding maps whose proof can be found in [49, 5, 1].

We say that $n$ is a $(\sigma, \delta, b)$-hyperbolic time of $f$ for a point $x$ if there are $0<\sigma<1$

and $b, \delta>0$ such that $\prod_{j=n-k}^{n-1}\left\|D f\left(f^{j}(x)\right)^{-1}\right\| \leq \sigma^{k}$ and $d_{\delta}\left(f^{k}(x), \delta\right) \geq e^{-b k}$ hold for all $k=0, \ldots, n-1$.

We now outline the properties of these special times. For detailed proofs see [5, Proposition 2.8] and [3, Proposition 2.6, Corollary 2.7, Proposition 5.2].

Proposition 5.3. There are constants $C_{1}, \delta_{1}>0$ depending on $(\sigma, \delta, b)$ and $f$ only such that, if $n$ is $(\sigma, \delta, b)$-hyperbolic time of $f$ for $x$, then there are hyperbolic pre-balls $V_{k}(x)$ which are neighborhoods of $f^{n-k}(x), k=1, \ldots, n$, satisfying

1. $f^{k} \mid V_{k}(x)$ maps $V_{k}(x)$ diffeomorphically to the ball of radius $\delta_{1}$ around $f^{n}(x)$; 
2. $d\left(f^{n-k}(y), f^{n-k}(z)\right) \leq \sigma^{k / 2} \cdot d\left(f^{n}(y), f^{n}(z)\right)$ for every $1 \leq k \leq n$ and $y, z \in V_{k}(x)$;

3. $C_{1}^{-1} \leq\left|\operatorname{det} D f^{n-k}(y)\right| /\left|\operatorname{det} D f^{n-k}(z)\right| \leq C_{1}$ for $y, z \in V_{k}(x)$.

The following ensures existence of infinitely many hyperbolic times for $\mu$-almost every point for non-uniformly expanding maps with respect to an ergodic invariant probability measure $\mu$. A complete proof can be found in [5, Section 5].

Theorem 5.4. Let $f: M \rightarrow M$ be a $C^{1+\alpha}$ local diffeomorphism away from a non-flat singular set $\mathcal{S}$, for some $\alpha \in(0,1)$, non-uniformly expanding and with slow recurrence to $\mathcal{S}$, with respect to an ergodic invariant probability measure $\mu$. That is there exists $c>0$ such that

$$
\limsup _{n \rightarrow+\infty} \frac{1}{n} S_{n} \psi \leq-c \quad \mu \text {-almost everywhere }
$$

and for every $\varepsilon>0$ there exists $\delta>0$ such that

$$
\limsup _{n \rightarrow \infty} \frac{1}{n} S_{n} \Delta_{\delta}(x) \leq \varepsilon \quad \mu \text {-almost everywhere. }
$$

Then there are $\sigma \in(0,1), \delta, b>0$ and there exists $\theta=\theta(\sigma, \delta, b)>0$ such that $\mu$-a.e. $x \in M$ has infinitely many $(\sigma, \delta, b)$-hyperbolic times. Moreover if we write $0<n_{1}<n_{2}<n_{2}<\ldots$ for

the hyperbolic times of $x$ then their asymptotic frequency satisfies $\lim _{\inf _{N \rightarrow \infty}} \frac{\#\left\{k \geq 1: n_{k} \leq N\right\}}{N} \geq \theta$ for Leb-a.e. $x \in M$.

\subsection{Existence of generating partition}

Let $\mu$ be an $f$-invariant ergodic probability measure in the conditions of the first part of the statement of Theorem 5.1.

Observe first that since $\mu(\psi)<0$ and $\mu$ is ergodic, then $f$ is non-uniformly expanding. Moreover by the assumptions on $\mu\left(\Delta_{\delta}\right)$ we see that $f$ has also slow recurrence to $\mathcal{S}$ with respect to $\mu$. Hence by Theorem 5.4 there are $\sigma, \delta, b>0$ such that $\mu$-almost all $x \in M$ admits infinitely many $(\sigma, \delta, b)$-hyperbolic times with positive frequency at infinity. Thus there exists a finite partition $\mathcal{P}_{0}$ of $M$ which is generating with respect to $\mu$.

Indeed let $\mathcal{E}=\left\{B\left(x_{i}, \delta_{1} / 8\right), i=1, \ldots, l\right\}$ be a finite open cover of $M$ by $\delta_{1} / 8$-balls whose boundary has zero $\mu$ measure. From this we define a finite partition $\mathcal{P}_{0}$ of $M$ as follows. Start by setting $P_{1}=B\left(x_{1}, \delta_{1} / 8\right)$ as the first element of the partition. Then, assuming that $P_{1}, \ldots, P_{k}$ are already defined, set $P_{k+1}=B\left(x_{k+1}, \delta_{1} / 8\right) \backslash\left(P_{1} \cup \cdots \cup P_{k}\right)$ for $k=1, \ldots, l-1$. Note that if $P_{k} \neq \emptyset$ then $P_{k}$ has non-empty interior, diameter smaller than $\delta_{1} / 4$ and the boundary $\partial P_{k}$ is a (finite) union of pieces of boundaries of balls in a Riemannian manifold. Thus $\partial P_{k}$ has zero Lebesgue measure and zero $\mu$-measure also. Define $\mathcal{P}_{0}$ by the elements $P_{k}$ constructed above which are non-empty. Note that $\mu\left(\partial \mathcal{P}_{0}\right)=\operatorname{Leb}\left(\partial \mathcal{P}_{0}\right)=0$ and by the existence of infinitely many $(\sigma, \delta, b)$-hyperbolic times for $\mu$-almost every $x$ it is not difficult to see that $\operatorname{diam}\left(\bigvee_{j=0}^{n-1} f^{-j} \mathcal{P}_{0}(x)\right) \underset{n \rightarrow+\infty}{\longrightarrow} 0$. 
Therefore, since $\mu$ satisfies the Entropy Formula, we can write

$$
\frac{1}{n} \int S_{n} \log J d \mu=\mu(\log J)=h_{\mu}(f)=h_{\mu}\left(f, \mathcal{P}_{0}\right) \leq \frac{1}{n} H_{\mu}\left(\mathcal{P}_{n}\right)=\frac{1}{n} \int-\log \mu\left(\mathcal{P}_{n}(x)\right) d \mu
$$

where $\mathcal{P}_{n}=\bigvee_{j=0}^{n-1} f^{-j} \mathcal{P}_{0}$ for $n \geq 1$. Hence by Jensen's Inequality we get, denoting $J_{n}(x)=\prod_{j=0}^{n-1} J\left(f^{j}(x)\right)$

$$
0 \geq \int \log \left[J_{n}(x) \cdot \mu\left(\mathcal{P}_{n}(x)\right)\right] d \mu(x) \geq \log \int J_{n}(x) \cdot \mu\left(\mathcal{P}_{n}(x)\right) d \mu(x) .
$$

If we define $Q_{\gamma}^{n}=\left\{x \in M: S_{n} J(x) \cdot \mu\left(\mathcal{P}_{n}(x)\right)>\gamma\right\}$ we obtain

$$
\mu\left(Q_{\gamma}^{n}\right) \leq \gamma^{-1} \text { for all } n \geq 1 .
$$

Now choose $\gamma_{n}>0$ such that $\sum_{n} \gamma_{n}^{-1}<\infty$. Then for $\mu$-almost every $x \in M$ there exists $n_{0} \in \mathbb{N}$ such that for all $n \geq n_{0}$ we have $x \notin Q_{\gamma_{n}}^{n}$, i.e. $J_{n}(x) \cdot \mu\left(\mathcal{P}_{n}(x)\right) \leq \gamma_{n}$ for all $n \geq n_{0}=n_{0}(x)$. Observe that by the definition and properties of hyperbolic times, we have that there exists $C_{1}>0$ such that

$$
C_{1}^{-1} \cdot \operatorname{Leb}\left(\mathcal{P}_{0}\left(f^{n}(x)\right)\right) \leq \operatorname{Leb}\left(\mathcal{P}_{n}(x)\right) \cdot J_{n}(x) \leq C_{1} \cdot \operatorname{Leb}\left(\mathcal{P}_{0}\left(f^{n}(x)\right)\right)
$$

whenever $n$ is a hyperbolic time for $x$. This shows that the $\mu$-measure of the atoms of $\mathcal{P}_{n}$ can be bounded from above by the volume of the same atoms at big enough hyperbolic times

$$
\mu\left(\mathcal{P}_{n}(x)\right) \leq C_{0} \gamma_{n} \operatorname{Leb}\left(\mathcal{P}_{n}(x)\right),
$$

where $C_{0}=C_{1} \sup _{x \in M} \operatorname{Leb}\left(\mathcal{P}_{0}(x)\right)$. The hyperbolic times satisfying this condition will be called $\mu$-hyperbolic times. To use this we need some way to cover any set using atoms of the sequence $\left(\mathcal{P}_{n}\right)_{n}$ at $\mu$-hyperbolic times.

\subsection{Coverings by hyperbolic times}

Let $\mu, f$ and $\left(\mathcal{P}_{n}\right)_{n \geq 0}$ be as in the previous subsection. Note that since $f$ is regular and $\mu$ is $f$-invariant the boundary of $g(P)$ still has zero Lebesgue measure and zero $\mu$-measure for every atom $P \in \mathcal{P}_{0}$ and every inverse branch $g$ of $f^{n}$, for any $n \geq 1$.

We can now state the following flexible covering lemma with $\mu$-hyperbolic preballs. It will enable us to approximate the $\mu$-measure of a given set through the measure of families of $\mu$-hyperbolic preballs.

Lemma 5.5 (The Hyperbolic Covering Lemma). Let a measurable set $E \subset M, m \geq 1$ and $\zeta>0$ be given with $\mu(E)>0$. Let $\theta>0$ be a lower bound for the density of $\mu$-hyperbolic times for $\mu$-almost every point. Then there are integers $m<n_{1}<\cdots<n_{k}$ for $k=k(\zeta) \geq 1$ and families $\mathcal{E}_{i}$ of subsets of $M, i=1, \ldots, k$ such that 
1. $\varepsilon_{1} \cup \cdots \cup \mathcal{E}_{k}$ is a finite pairwise disjoint family of subsets of $M$;

2. $n_{i}$ is a $(\sigma / 2, \delta / 2)$ - $\mu$-hyperbolic time for every point in $P$, for every element $P \in \mathcal{E}_{i}, i=$ $1, \ldots, k$;

3. every $P \in \mathcal{E}_{i}$ is the preimage of some element $Q \in \mathcal{P}$ under an inverse branch of $f^{n_{i}}$, $i=1, \ldots, k$;

4. there is an open set $U_{1} \supset E$ containing the elements of $\varepsilon_{1} \cup \cdots \cup \varepsilon_{k}$ with $\mu\left(U_{1} \backslash E\right)<\zeta$;

5. $\mu\left(E \Delta \bigcup_{i} \varepsilon_{i}\right) \leq\left(1-\frac{\theta}{4}\right)^{k}<\zeta$.

The proof is completely presented in [7, Lemma 3.5] and follows [47, Lemma 8.2] closely.

\subsection{Absolute continuity}

We are now ready to deduce that any measure $\mu$ as in the statement of Theorem 5.1 is absolutely continuous. Indeed observe that, by (5.1) and the choice of $\left(\gamma_{n}\right)_{n \geq 1}$, for any given $\eta>0$ we can find $N=N(\eta) \in \mathbb{N}$ such that $\Gamma_{\eta}=\cap_{n \geq N}\left(M \backslash Q_{\gamma_{n}}^{n}\right)$ satisfies $\mu\left(\Gamma_{\eta}\right) \geq 1-\eta$.

Let $E \subset M$ be given with $\mu\left(E \cap \Gamma_{\eta}\right)>0$. Let $m=N$ in the statement of the Covering Lemma[5.5] and set $\zeta>0$ small. Then we get $\mu\left(\left(E \cap \Gamma_{\eta}\right) \Delta \cup_{i=1}^{k} \varepsilon_{i}\right)<\zeta$ where all elements of $\mathcal{E}_{i}$ are $\mu$-preballs and atoms of $\mathcal{P}_{n_{i}}$ satisfying the bound (5.2). In particular by the choice of $m$ we have $\cup_{i} \mathcal{E}_{i} \subset \Gamma_{\eta}$ and so we may write

$$
\mu(E)=\mu\left(E \cap M \backslash \Gamma_{\eta}\right)+\mu\left(E \cap \Gamma_{\eta}\right) \leq \eta+\zeta+\mu\left(E \cap \cup_{i} \varepsilon_{i}\right) \leq \eta+\zeta+C_{0} \gamma_{n_{k}} \operatorname{Leb}\left(E \cap \cup_{i} \varepsilon_{i}\right),
$$

where $n_{k}$ is the largest $\mu$-hyperbolic time used in the cover given by the Hyperbolic Covering Lemma.

Hence if we start with a subset $E$ with $\operatorname{Leb}(E)=0$ and assume that $\mu(E)>0$, then there exists $\eta_{0}$ such that $\mu\left(E \cap \Gamma_{\eta}\right)>0$ for all $0<\eta \leq \eta_{0}$. Therefore given $\zeta>0$ as above we obtain (5.3). But since $\operatorname{Leb}(E)=0$ we get $\mu(Z) \leq \eta+\zeta$, for all $0<\eta \leq \eta_{0}$, that is $\mu(Z) \leq \zeta$. This is a contradiction since we may take $\zeta>0$ as small as we like.

We have shown that if $\operatorname{Leb}(E)=0$ then $\mu(E)=0$, i.e. $\mu \ll$ Leb. Then since the basins of the physical measures of $f$ cover $M$ except for a volume zero subset, then it follows easily by the Ergodic Theorem that $\mu=\sum_{i=1}^{k} \mu\left(B\left(\mu_{i}\right)\right) \cdot \mu_{i}$, that is $\mu \in \overline{\mathrm{co}}(\mathbb{F})$.

Reciprocally, let us now assume that $\mu$ is an $f$-invariant absolutely continuous probability measure. Then as above we have $\mu \in \overline{\mathrm{co}}(\mathbb{F})$ and and thus for some constants $\alpha_{i} \geq 0$ such that $\sum_{i} \alpha_{i}=1$ we have $h_{\mu}(f)=\sum_{i=1}^{k} \alpha_{i} h_{\mu_{i}}(f)=\sum_{i=1}^{k} \alpha_{i} \mu_{i}(\log J)=\mu(\log J)$. This concludes the proof of Theorem 5.1. 


\section{Exponentially slow approximation to singularities}

Here we apply the (by now standard) arguments of Benedicks and Carleson, first presented in [16, 17], to show that Lorenz-like maps have exponentially slow recurrence to singularities. This completes the presentation of the examples in Section 2.2.

Let $f: M \rightarrow M$ be a one-dimensional $C^{1+\alpha}$ piecewise expanding map with at most countably many smoothness domains for some $\alpha \in(0,1)$ as in Subsection 2.2. that is $\left|f^{\prime}\right| \geq \sigma>1$ and the non-degenerate singular set $\mathcal{S}$ equals the boundaries of the smoothness domains and satisfies all the conditions (S1) through (S5). Then $\mathcal{S}=\left\{b_{n}\right\}_{n}$ where we may assume that the sequence is strictly monotonous (in counter-clockwise order if $M=\mathbb{S}^{1}$ ).

We consider the middle points $c_{n}=\left(b_{n}+b_{n+1}\right) / 2$ for all applicable indexes $n$ to define a Lebesgue modulo zero partition $\mathcal{P}_{0}$ of $M$ as follows.

\subsection{Initial partition}

Partition $\left(b_{n}, c_{n}\right)$ into subintervals

$$
M(2 n, p)=\left(b_{n}+d_{2 n} e^{-p}, b_{n}+d_{2 n} e^{-(p-1)}\right),
$$

where $d_{2 n}=c_{n}-b_{n}$ and partition the interval $\left(c_{n-1}, b_{n}\right)$ into the following subintervals

$$
M(2 n-1, p)=\left(b_{n}-d_{2 n-1} e^{-(p-1)}, b_{n}-d_{2 n-1} e^{-p}\right)
$$

where $d_{2 n-1}=b_{n}-c_{n-1}$, for all $p \geq 1$. The sets defined above form a partition of $M$ Lebesgue modulo zero consisting of small intervals whose length is exponentially small with respect to the distance to $\mathcal{S}$. Let $\mathcal{S}^{\prime}=\mathcal{S} \cap f(M)$ be the set of singular points of $f$ which matter for the asymptotic dynamics of $f$.

To define the initial partition consider a threshold $\rho_{0} \in \mathbb{N}$ such that

$$
e^{-\beta \rho_{0}}<1 \text { and }\left(1+\frac{2}{\rho_{0}}\right)\left(1+\frac{\rho_{0}}{2}\right)^{2 / \rho_{0}}<e^{\beta}
$$

and let $\mathcal{P}_{0}$ be formed by the collection of all intervals $M(n, p)$ for all $n$ and every $p \geq \rho_{0}$ together with the connected components of $M \backslash\left(\cup_{n ; p \geq \rho_{0}} M(n, p) \cup\left\{c_{n}\right\}_{n}\right)$, which will be denoted by $M\left(n, \rho_{0}-1\right)$ whenever they are adjacent to $M\left(n, \rho_{0}\right)$.

For each element $\eta$ of $\mathcal{P}_{0}$ denote by $\eta^{+}$the interval obtained by joining $\eta$ with its two neighboring intervals in $\mathcal{P}_{0}$. From (6.1) and (6.2) we have the following relations for all $k$ and every $p \geq \rho_{0}-1$

$$
\operatorname{Leb}\left(M(k, p)^{+}\right) \leq 9 \operatorname{Leb}(M(k, p))=9 d_{k} \cdot e^{-p}(e-1) .
$$




\subsection{Refining the partition}

The partition $\mathcal{P}_{0}$ is dynamically refined so that any pair $x, y$ of points in the same atom of the $n$th refinement $\mathcal{P}_{n}$ belong to the same element of $\mathcal{P}_{0}$ during the first consecutive $n$ iterates, i.e. $\mathcal{P}_{0}\left(f^{i}(x)\right)=\mathcal{P}_{0}\left(f^{i}(y)\right)$ for $i=0, \ldots, n-1$. Moreover $f^{n} \mid \omega$ is a diffeomorphism for every interval $\omega \in \mathcal{P}_{n}$.

The refinement is defined inductively. Assume that $\mathcal{P}_{n}$ is already defined and for each $\omega \in \mathcal{P}_{n}$ there are sets $R_{n}(\omega)$ of splitting times and $D_{n}(\omega)$ of corresponding splitting depths, to be defined below.

If $f^{n+1}(\omega)$ intersects three or fewer elements of $\mathcal{P}_{0}$, then we set $\omega \in \mathcal{P}_{n+1}, R_{n+1}(\omega)=$ $R_{n}(\omega)$ and $D_{n+1}(\omega)=D_{n}(\omega)$. Otherwise consider the subsets $\eta^{\prime}=\left(f^{n+1} \mid \omega\right)^{-1}(\eta)$ of the interval $\omega$, for all elements $\eta$ of $\mathcal{P}_{0}$ which intersect $f^{n+1}(\omega)$.

The family $\left\{\eta^{\prime}\right\}$ obtained above is a partition of $\omega$. Observe that $f^{n+1}\left(\eta^{\prime}\right)$ is either equal to some $\eta \in \mathcal{P}_{0}$ or strictly contained in some $\eta \in \mathcal{P}_{0}$. In the latter case we redefine the partition joining some of the extreme intervals of $\left\{\eta^{\prime}\right\}$ with its neighbors so that the new partition $\{\zeta\}$ of $\omega$ satisfies: for each $\zeta$ there exists $\eta=M(k, p) \in \mathcal{P}_{0}$ such that $\eta \subseteq f^{n}(\zeta) \subseteq \eta^{+}$.

Finally we set $\zeta \in \mathcal{P}_{n+1}, R_{n+1}(\zeta)=R_{n}(\zeta) \cup\{n+1\}$ and $D_{n+1}(\zeta)=D_{n}(\zeta) \cup\{(k, p)\}$, for each element of the partition $\{\zeta\}$ of $\omega$ constructed above. For these elements of $\mathcal{P}_{n+1}$ we say that $n+1$ is a splitting time and the pairs $(k, p)$ are the corresponding splitting depths. Repeat the procedure for each $\omega \in \mathcal{P}_{n}$. This completes the construction of $\mathcal{P}_{n+1}$ from $\mathcal{P}_{n}$ for all $n \geq 0$.

\subsection{Bounded distortion}

The uniform expansion of length during $n$ iterates ensures that we have bounded distortion of lengths on atoms of the partition $\mathcal{P}_{n}$.

Indeed let $\omega \in \mathcal{P}_{n}$ for some $n \geq 1$ and let $x, y \in \omega$. Note that $f^{i} \mid \omega$ is a diffeomorphism for $i=1, \ldots, n, f$ expands distances at a minimum rate of $\sigma$ and $f^{\prime}$ is $\alpha$-Hölder. Then there exist constants $C, D>0$ such that

$$
\begin{aligned}
\log \left|\frac{\left(f^{n}\right)^{\prime}(x)}{\left(f^{n}\right)^{\prime}(y)}\right| & =\sum_{i=0}^{n-1}|\log | f^{\prime}\left(f^{i}(x)\right)|-\log | f^{\prime}\left(f^{j}(y) \mid \leq \sum_{i=0}^{n-1} C \cdot \frac{\left|f^{i}(x)-f^{j}(y)\right|^{\alpha}}{\max \left\{\left|f^{\prime}\left(f^{i}(x)\right)\right|,\left|f^{\prime}\left(f^{i}(y)\right)\right|\right\}}\right. \\
& \leq \frac{C}{\sigma} \sum_{i=0}^{n-1} \sigma^{i-n} \cdot\left|f^{n}(x)-f^{n}(y)\right|^{\alpha} \leq D
\end{aligned}
$$

where $D$ depends only on $\sigma$ and on the diameter of $M$.

\subsection{Measure of atoms of $\mathcal{P}_{n}$ and return depths}

Here we show that we can estimate the measure of an element of $\mathcal{P}_{n}$ using the information stored in $R_{n}$ and $D_{n}$.

For any given $n \geq 1$ and $\omega \in \mathcal{P}_{n}$ we have 
- a sequence of times $R_{n}(\omega)=\left\{r_{1}<\cdots<r_{s}\right\}$ with $r_{1} \geq 1$ and $r_{s} \leq n$, and

- a sequence of intervals $\omega_{0} \supsetneq \omega_{1} \supsetneq \cdots \supsetneq \omega_{s}=\omega$ with corresponding depths $D_{n}(\omega)=\left\{\left(k_{1}, p_{1}\right), \ldots,\left(k_{s}, p_{s}\right)\right\}$, where $\omega_{0} \in \mathcal{P}_{0}$ and $\omega_{i} \in \mathcal{P}_{r_{i}} \cap \cdots \cap \mathcal{P}_{r_{i+1}-1}$

such that

$$
M\left(k_{i}, p_{i}\right) \subseteq f^{r_{i}}\left(\omega_{i-1}\right) \subseteq M\left(k_{i}, p_{i}\right)^{+}
$$

for all $i=0,1, \ldots, s-1$ with $r_{0}=0$ and $s_{0}=0$. These times are the iterates where the images of the previous element of the partition was broken into smaller intervals as in Subsection 6.2. Using the bounded distortion given by (6.5) we get

$$
\frac{\operatorname{Leb}(\omega)}{\operatorname{Leb}\left(\omega_{0}\right)}=\frac{\operatorname{Leb}\left(w_{s}\right)}{\operatorname{Leb}\left(\omega_{s-1}\right)} \cdots \frac{\operatorname{Leb}\left(\omega_{1}\right)}{\operatorname{Leb}\left(\omega_{0}\right)} \leq \prod_{i=1}^{s} D \frac{\operatorname{Leb}\left(f^{r_{i}}\left(\omega_{i}\right)\right)}{\operatorname{Leb}\left(f^{r_{i}}\left(\omega_{i-1}\right)\right)} .
$$

Now using (6.6) and (S5) we bound the last expression from above by

$$
\prod_{i=1}^{s} \frac{D \operatorname{Leb}\left(M\left(k_{i}, p_{i}\right)^{+}\right)}{B^{-1} e^{\beta p_{i-1}} d_{k_{i-1}}^{-\beta}(e-1)^{-\beta} \operatorname{Leb}\left(M\left(k_{i-1}, p_{i-1}\right)\right)}
$$

and using (6.4) this can be easily simplified yielding

$$
\operatorname{Leb}(\omega) \leq \prod_{i=0}^{s-1} d_{k_{i}}^{\beta} e^{-2 \beta p_{i}} \leq \exp \left(-\beta \sum_{i=0}^{s-1}\left(p_{i}+q_{i}\right)\right)
$$

where $q_{i}=\left[-\log d_{k_{i}}\right]$ with $[z]=\max \{k \in \mathbb{Z}: k \leq z\}$. We have used $p \geq \rho_{0}$ and $\log \left(9 B D(e-1)^{\beta}\right) / \rho_{0} \leq \beta$ to compensate the constants on the exponent $2 \beta$. Recall also that $\omega_{0}=M\left(k_{0}, p_{0}\right)$. Note also that if $R_{n}(\omega)=\emptyset$, then since there is no splitting but there is uniform expansion together with distortion control, we get

$$
\operatorname{Leb}\left(f^{n}(\omega)\right)=\int_{\omega}\left|\left(f^{n}\right)\right| d \operatorname{Leb} \geq D \sigma^{n} \operatorname{Leb}(\omega) \text { so } \operatorname{Leb}(\omega) \leq D^{-1} \sigma^{-n} .
$$

\subsection{Distance to $\mathcal{S}$ and splitting depths}

Let again $n \geq 1$ and $\omega \in \mathcal{P}_{n}$ be given and consider the sets $R_{n}(\omega)$ and $D_{n}(\omega)$. Consider the intervals $\omega_{0} \supsetneq \omega_{1} \supsetneq \cdots \supsetneq \omega_{s}=\omega$ as before. Note that for the iterates $i$ between two consecutive times $r<r^{\prime}$ from $R_{n}$, i.e. if $r<i<r^{\prime}$ then there exists $M\left(l_{i}, q_{i}\right) \in \mathcal{P}_{0}$ such that $f^{i}\left(\omega_{r}\right) \subseteq M\left(l_{i}, q_{i}\right)^{+}$by this choice of $i$. Moreover by condition (S5) and by (6.1) and (6.2) we deduce

$$
\begin{aligned}
9 d_{l_{1}}(e-1) e^{-q_{1}} & \geq \operatorname{Leb}\left(f^{r+1}\left(\omega_{r}\right)\right) \geq\left(B d_{k_{r}} e^{-p_{r}}\right)^{-\beta} \operatorname{Leb}\left(f^{r}\left(\omega_{r}\right)\right) \\
& =\left(\frac{B}{e-1} \operatorname{Leb}\left(f^{r}\left(\omega_{r}\right)\right)\right)^{-\beta} \operatorname{Leb}\left(f^{r}\left(\omega_{r}\right)\right) \\
& =\left(\frac{e-1}{B}\right)^{\beta} \operatorname{Leb}\left(f^{r}\left(\omega_{r}\right)\right)^{1-\beta} .
\end{aligned}
$$


Hence $d_{l_{1}} e^{-q_{1}-1} \geq(9 e(e-1))^{-1} \operatorname{Leb}\left(f^{r+1}\left(\omega_{r}\right)\right)$ is the estimate for the minimum distance from $\mathcal{S}$ to $f^{r+1}\left(\omega_{r}\right)$. Let $L_{i}=\operatorname{Leb}\left(f^{r+i}\left(\omega_{r}\right)\right)$ and $D_{i}=\operatorname{dist}\left(f^{r+i}\left(\omega_{r}\right), \mathcal{S}\right)$ for $i=0, \ldots, r^{\prime}-r-1$. Then the reasoning above shows that $L_{1} \geq\left(\frac{e-1}{B}\right)^{\beta} L_{0}^{1-\beta}$ and $L_{i+1} \geq\left(\frac{9 e(e-1)}{B}\right)^{\beta} L_{i}^{1-\beta}$, and also $D_{i} \geq L_{i} /(9 e(e-1))$ for $i=1, \ldots, r^{\prime}-r-1$. It is now easy to see that

$$
\begin{aligned}
-\log L_{i+1} & \leq-(1-\beta) \log L_{i}+\beta \log \frac{9 e(e-1)}{B} \\
& =-\left(1-\beta-\beta \log \left(\frac{9 e(e-1)}{B}\right) / \log L_{i}\right) \log L_{i}=-\gamma \log L_{i}
\end{aligned}
$$

where we may assume that $\gamma \in(0,1)$ since it is no restriction to increase the value of $B$ if needed. Hence

$$
\begin{aligned}
-\sum_{i=1}^{r^{\prime}-r-1} \log \operatorname{dist}\left(f^{r+i}\left(\omega_{r}\right), \delta\right) & \leq-\sum_{i=1}^{r^{\prime}-r-1}\left(\log L_{i}-\log (9 e(e-1))\right) \\
& \leq- \text { Const } \cdot \log L_{0}+\left(r^{\prime}-r\right) \log (9 e(e-1)) \\
& \leq- \text { Const } \cdot \log L_{0},
\end{aligned}
$$

since by uniform expansion and by definition of $r^{\prime}$ we have $\sigma^{r^{\prime}-r} L_{0} \leq 1$ and also $r^{\prime}-r \leq$ $-\log \left(L_{0}\right) / \log \sigma$. Since $r<r^{\prime}$ were two arbitrary consecutive elements of $R_{n}(\omega)$ for $\omega \in \mathcal{P}_{n}$ we have shown that

$$
\sum_{j=0}^{s-1}-\log \operatorname{dist}\left(f^{j}(x), s\right) \leq- \text { Const } \sum_{(k, p) \in D_{s}(\omega)} \log \left(d_{k} e^{-p}\right)
$$

for all $x \in \omega$, where $s<n$ is the last splitting time before $n\left(s=\max R_{n}(\omega)\right)$.

However if $m>n$ is the first integer such that $\omega \notin \mathcal{P}_{m}$ but $\omega \in \mathcal{P}_{l}$ for $n<l<m$, then we can write the following disjoint union $\omega=\bigcup_{\omega^{\prime} \in \mathcal{P}_{m}} \omega^{\prime} \cap \omega$. Repeating the argument for $x \in \omega^{\prime} \cap \omega$ for each $\omega^{\prime} \in \mathcal{P}_{m}$ intersecting $\omega$ we can obtain a relation like (6.9) with $D_{s}(\omega)$ replaced by $D_{n}(\omega)$ as the summation range, where $n$ is between $s$ and $m$. This shows that the average of the log of the distance to the singular set is bounded by the sum of the depths at splitting times modulo a constant.

\subsection{Expected value of splitting depths}

Now we estimate the expected value of the splitting depths for deep splitting times up to $n$ iterates of the map. Define for a co-countable set of $x \in M$ the function $\mathcal{D}_{n}(x)=$ $-\sum_{(k, p) \in D_{n}\left(\mathcal{P}_{n}(x)\right)} \log \left(d_{k} e^{-p}\right)$ where $\mathcal{P}_{n}(x)$ is the unique atom of $\mathcal{P}_{n}$ which contains $x \in M$. Define also the truncated sum: for any given $\delta>0$ set for the same points $x \in M$ as above

$$
\mathcal{D}_{n}^{\delta}(x)=\sum_{\substack{(k, p) \in D_{n}\left(\mathcal{P}_{n}(x)\right) \\ d_{k} e^{-p}<\delta}}-\log \left(d_{k} e^{-p}\right) .
$$


By the arguments in Subsection 6.5 and by the definitions (6.1) and (6.2) we obtain

$$
\sum_{j=0}^{n-1}-\log \operatorname{dist}_{\delta}\left(f^{j}(x), \mathcal{S}\right) \leq \mathcal{D}_{n}^{\delta}(x) .
$$

Define the number of splittings up to the nth iterate $t_{n}(x)=\# R_{n}\left(\mathcal{P}_{n}(\omega)\right)$ and also the number of deep splittings among these $u_{n}(x)=\#\left\{(k, p) \in R_{n}\left(\mathcal{P}_{n}(\omega)\right): d_{k} e^{-p}<\delta\right\}$.

Given $x$ and $n \geq 1$ we let $0=r_{0}<r_{1}<\cdots<r_{t}$ with $t=t_{n}(x)$ be the splitting times along the orbit of $x$ up to the $n$th iterate and $0 \leq s_{1}<\cdots<s_{u}$ be indexes corresponding to deep splittings, where $u=u_{n}(x)$ in what follows. Note that each quantity above is constant on the elements of $\mathcal{P}_{n}$. Define

$$
A_{\left(\kappa_{1}, \rho_{1}\right), \ldots,\left(\kappa_{u}, \rho_{u}\right)}^{u, t}(n)=\left\{x \in M: t_{n}(x)=t, u_{n}(x)=n \text { and }\left(k_{s_{i}}, p_{s_{i}}\right)=\left(\kappa_{i}, \rho_{i}\right), i=1, \ldots, u\right\}
$$

the set of points which in $n$ iterates have $t$ splitting times and $u$ deep splittings among these, with the specified depths $\left(\kappa_{1}, \rho_{1}\right), \ldots,\left(\kappa_{u}, \rho_{u}\right)$.

Lemma 6.1. $\operatorname{Leb}\left(A_{\left(\kappa_{1}, \rho_{1}\right), \ldots,\left(\kappa_{u}, \rho_{u}\right)}^{u, t}(n)\right) \leq\left(\begin{array}{l}t \\ u\end{array}\right) \exp \left(-\beta \sum_{i=1}^{u}\left(\eta_{i}+\rho_{i}\right)\right)$ where $\eta_{i}=\left[-\log d_{\kappa_{i}}\right]$.

Proof. Using the estimate (6.7) we get the following bound for the Lebesgue measure of $A_{\left(\kappa_{1}, \rho_{1}\right) \ldots, \ldots,\left(\kappa_{u}, \rho_{u}\right)}^{u, t}(n)$

$$
\left(\begin{array}{l}
t \\
u
\end{array}\right) \exp \left(-\beta \sum_{i=1}^{u}\left(\eta_{i}+\rho_{i}\right)\right) \cdot \exp \left(-\beta \sum_{\substack{\left(k_{j}, p_{j}\right) \\
j=1, \ldots, d_{k} e^{-p_{j}} \geq \delta}}\left(v_{j}+p_{j}\right)\right) .
$$

The binomial coefficient takes into account all the possible orderings of sequences of $u$ deep splitting times among $t$ splitting times and the last exponential bounds the contribution of all the possible $t-u$ non-deep splitting times, with $v_{j}=\left[-\log d_{k_{j}}\right]$. But since $p \geq \rho_{0}$ was chosen as in (6.3) and $v_{j} \geq 0$ we conclude that the last exponential is smaller than 1 . So we obtain the bound in the statement.

Lemma 6.2. For any $z>\beta$ we have $\int e^{z \mathcal{D}_{n}^{\delta}(x)} d x \leq e^{\theta(\delta) n}$ where $\theta(\delta)$ is such that $\theta(\delta) \searrow 0$ when $\delta \searrow 0$.

Proof. By definition

$$
\begin{aligned}
\int e^{z \mathcal{D}_{n}^{\delta}(x)} d x= & \sum_{\omega \in \mathcal{P}_{n}} e^{z \mathcal{D}_{n}^{\delta}(\omega)} \cdot \operatorname{Leb}(\omega) \leq \sum_{\substack{\omega_{0} \in \mathcal{P}_{0} \\
D \sigma^{n} \operatorname{Leb}\left(\omega_{0}\right) \leq 1}} \operatorname{Leb}\left(\omega_{0}\right) \\
& +\sum_{0<u \leq t<n} \sum_{\substack{\left(\kappa_{i}, \rho_{i}\right) \\
i=1, \ldots, u}} e^{z \mathcal{D}_{n}^{\delta}(\omega)} \operatorname{Leb}\left(A_{\left(\kappa_{1}, \rho_{1}\right), \ldots,\left(\kappa_{u}, \rho_{u}\right)}^{u, t}(n)\right)
\end{aligned}
$$


where we are considering all possible combinations of splitting depths and of deep splittings among all the splitting times, for all elements of $\mathcal{P}_{n}$ in the second sum.

Consider the first term corresponding to the atoms of $\mathcal{P}_{0}$ which were not split during the first $n$ iterates. This sum can be separated as follows

$$
\sum_{\substack{\omega_{0} \in \mathcal{P}_{0} \\ D \sigma^{n} \operatorname{Leb}\left(\omega_{0}\right) \leq 1}} \operatorname{Leb}\left(\omega_{0}\right)=\sum_{\substack{D \sigma^{n} \operatorname{Leb}\left(\omega_{0}\right) \leq 1 \\ d_{k} \sigma^{n / 2}<1}} \operatorname{Leb}\left(\omega_{0}\right)+\sum_{\substack{D \sigma^{n} \operatorname{Leb}\left(\omega_{0}\right) \leq 1 \\ d_{k} \sigma^{n / 2} \geq 1}} \operatorname{Leb}\left(\omega_{0}\right) \leq \operatorname{Leb}\left(B\left(\mathcal{S}, \sigma^{-n / 2}\right)\right)+\sum_{\substack{ \\p>\log \left(D(e-1) \sigma^{n / 2}\right)}} e^{-p} \leq C e^{-c n}
$$

for some constants $C, c>0$, where we have used expression (6.4) for the length of the atoms of $\mathcal{P}_{0}$ in terms of $(k, p)$ together with condition (S4) and the obvious $d_{k}>0$ and $\sum_{k} d_{k}=1$. Note that if $\mathcal{S}$ is finite then the condition $d_{k} \sigma^{n / 2}<1$ is always false for big enough $n$. So in this case we only have the right hand side sum above.

Now we bound the second term (6.12). Considering Lemma 6.1 and taking into account $\mathcal{D}_{n}^{\delta}$ we obtain (with $\eta_{j}=\left[-\log d_{\kappa_{j}}\right]$ )

$$
\sum_{0<u \leq t<n} \sum_{\substack{\left(\kappa_{i}, \rho_{i}\right) \\
i=1, \ldots, u}}\left(\begin{array}{l}
t \\
u
\end{array}\right) e^{-(\beta+z) \sum_{i}\left(\eta_{i}+\rho_{i}\right)} \leq \sum_{0<u \leq t<n} \sum_{h>u \ell(\delta)}\left(\begin{array}{l}
t \\
u
\end{array}\right) u L(h, u) e^{-(\beta+z) h}
$$

where $h=\sum_{i}\left(\eta_{i}+\rho_{i}\right), \ell(\delta)$ is an integer such that every pair $(k, p)$ satisfying $d_{k} e^{-p}<\delta$ also satisfies $k+p>\ell(\delta)$, and

$$
L(h, u)=\#\left\{\left(\left(\eta_{i}, \rho_{i}\right)\right)_{i=1, \ldots, u} \in \mathbb{N}_{0}^{2 u}: \sum_{i=1}^{u}\left(\eta_{i}+\rho_{i}\right)=h \text { with } \rho_{i} \geq \rho_{0}\right\} .
$$

Moreover the factor $u$ bounds the number of distinct $d_{k_{i}}$ with the same value $\eta_{i}$ along the $n$ iterates of the orbit of the points. Observe that

$$
L(h, u) \leq \#\left\{\left(h_{i}\right) \in \mathbb{N}_{0}^{2 u}: \sum_{i=0}^{2 u} h_{i}=h\right\}=\left(\begin{array}{c}
h+2 u-1 \\
2 u-1
\end{array}\right)
$$

and by a standard application of Stirling's Formula

$$
L(h, n) \leq\left(c^{1 / h}\left(1+\frac{2 u-1}{h}\right)\left(1+\frac{h}{2 u-1}\right)^{(2 u-1) / h}\right)^{h} \leq e^{\beta h} \leq e^{z h}
$$

where $0<c<1$ is a constant independent of the other variables and the last inequalities follow by $h \geq \rho_{0} u$, by the choice of $\rho_{0}$ in (6.3) and by taking $z>\beta$.

Collecting the bounds we have obtained we conclude that the second sum in (6.12) can be bounded by the following expression

$$
\begin{aligned}
\sum_{0<u \leq t<n}\left(\begin{array}{l}
t \\
u
\end{array}\right) u \sum_{h>u \ell(\delta)} e^{-\beta h} & \leq \sum_{u=0}^{n} n\left(\begin{array}{c}
n-1 \\
u
\end{array}\right) \cdot u e^{-\beta u \ell(\delta) / 2} \cdot \frac{e^{-\beta u \ell(\delta) / 2}}{1-e^{-\beta}} \leq \sum_{u=0}^{n}\left(\begin{array}{l}
n \\
u
\end{array}\right) C \frac{\left(e^{-\beta \ell(\delta) / 2}\right)^{u}}{1-e^{-\beta}} \\
& \leq\left(1+\frac{C}{1-e^{-\beta}} e^{-\beta \ell(\delta) / 2}\right)^{n}
\end{aligned}
$$


for some constant $C>0$ bounding $\left\{u e^{-\beta u \ell(\delta) / 2}\right\}_{u \geq 0}$ (which can be taken independently of $\ell(\delta)$ ). Finally since $\ell(\delta)$ grows without limit when $\delta \searrow 0$, the statement of the lemma follows just by increasing the value of $C$ to take into account the small bound of the first sum (6.13).

\subsection{Measure of the points with bad recurrence}

We are now ready to deduce exponentially slow approximation to the singular set $\mathcal{S}$. Indeed we just have to use Tchebishev's inequality, as follows: given $\varepsilon, \delta>0$ we know there exists a constant $C>0$ as in Subsection 6.5 such that

$$
\left\{x \in M:-\frac{1}{n} \sum_{i=0}^{n-1} \log \operatorname{dist}_{\delta}\left(f^{i}(x), \delta\right) \geq \varepsilon\right\} \subseteq\left\{x: \frac{\mathcal{D}_{n}^{\delta}(x)}{n} \geq \frac{\varepsilon}{C}\right\}=\left\{x: e^{z \mathcal{D}_{n}^{\delta}(x)} \geq e^{n \varepsilon / C}\right\}
$$

hence

$$
\operatorname{Leb}\left\{x \in M:-\frac{1}{n} \sum_{i=0}^{n-1} \log \operatorname{dist}_{\delta}\left(f^{i}(x), \delta\right) \geq \varepsilon\right\} \leq e^{-n \varepsilon / \mathcal{C}} \int e^{z \mathcal{D}_{n}^{\delta}} d \operatorname{Leb}=e^{-n(\varepsilon / \mathcal{C}-\theta(\delta))}
$$

which can be made exponentially small by choosing $\delta>0$ small enough so that $\varepsilon / C>\theta(\delta)$. This proves that a piecewise expanding map $f$ in our settings has exponentially slow recurrence to the singular set, completing the proof of the statements in Subsection 2.2 and of Corollary Cafter the reduction procedure of Subsection 2.4.

\section{References}

[1] J. Alves. Statistical analysis of non-uniformly expanding dynamical systems. Publicações Matemáticas do IMPA. [IMPA Mathematical Publications]. Instituto de Matemática Pura e Aplicada (IMPA), Rio de Janeiro, 2003. 24 Colóquio Brasileiro de Matemática. [24th Brazilian Mathematics Colloquium].

[2] J. F. Alves. SRB measures for non-hyperbolic systems with multidimensional expansion. Ann. Sci. École Norm. Sup., 33:1-32, 2000.

[3] J. F. Alves and V. Araujo. Random perturbations of nonuniformly expanding maps. Astérisque, 286:25-62, 2003.

[4] J. F. Alves and V. Araújo. Hyperbolic times: frequency versus integrability. Ergodic Theory and Dynamical Systems, 24:1-18, 2004.

[5] J. F. Alves, C. Bonatti, and M. Viana. SRB measures for partially hyperbolic systems whose central direction is mostly expanding. Invent. Math., 140(2):351-398, 2000. 
[6] J. F. Alves, S. Luzzatto, and V. Pinheiro. Markov structures and decay of correlations for non-uniformly expanding dynamical systems. Ann. Inst. H. Poincaré Anal. Non Linéaire, 22(6):817-839, 2005.

[7] V. Araújo and M. J. Pacifico. Large deviations for non-uniformly expanding maps. J. Stat. Phys., 125(2):415-457, 2006.

[8] V. Araujo and M. J. Pacifico. Physical measures for infinite-modal maps. Preprint IMPA Serie A, 328/2004.

[9] V. Araújo and A. Tahzibi. Stochastic stability at the boundary of expanding maps. Nonlinearity, 18:939-959, 2005.

[10] V. Araújo, E. R. Pujals, M. J. Pacifico, and M. Viana. Singular-hyperbolic attractors are chaotic. Preprint arxiv math.DS/0511352. To appear in Transactions of the A.M.S., 2007.

[11] A. Arbieto and C. Matheus. Fast decay of correlations of equilibrium states of open classes of non-uniformly expanding maps and potentials. Preprint http://arxiv.org/abs/math.DS/0603629, 2006.

[12] A. Arbieto, C. Matheus, S. Senti, and M. Viana. Maximal entropy measures for viana maps. Discrete and Continuous Dynamical Systems, to appear, 2007.

[13] A. Avila, S. Gouëzel, and J.-C. Yoccoz. Exponential mixing for the Teichmüller flow. Publ. Math. Inst. Hautes Études Sci., 104:143-211, 2006.

[14] J. Bahnmüller and P.-D. Liu. Characterization of measures satisfying the Pesin entropy formula for random dynamical systems. J. Dynam. Differential Equations, 10(3):425-448, 1998.

[15] V. Baladi and B. Vallée. Exponential decay of correlations for surface semi-flows without finite Markov partitions. Proc. Amer. Math. Soc., 133(3):865-874 (electronic), 2005.

[16] M. Benedicks and L. Carleson. On iterations of $1-a x^{2}$ on $(-1,1)$. Annals of Math., 122:1-25, 1985.

[17] M. Benedicks and L. Carleson. The dynamics of the Hénon map. Annals of Math., 133:73-169, 1991.

[18] M. Benedicks and L.-S. Young. Absolutely continuous invariant measures and random perturbations for certain one-dimensional maps. Ergod. Th. E Dynam. Sys., 12:13-37, 1992.

[19] M. Benedicks and L.-S. Young. SBR-measures for certain Hénon maps. Invent. Math., 112:541-576, 1993. 
[20] M. Benedicks and L.-S. Young. Markov extensions and decay of correlations for certain Hénon maps. Astérisque, 261:13-56, 2000.

[21] C. Bonatti, L. J. Díaz, and M. Viana. Dynamics beyond uniform hyperbolicity, volume 102 of Encyclopaedia of Mathematical Sciences. Springer-Verlag, Berlin, 2005. A global geometric and probabilistic perspective, Mathematical Physics, III.

[22] R. Bowen. Equilibrium states and the ergodic theory of Anosov diffeomorphisms, volume 470 of Lect. Notes in Math. Springer Verlag, 1975.

[23] R. Bowen and D. Ruelle. The ergodic theory of Axiom A flows. Invent. Math., 29:181-202, 1975.

[24] H. Bruin and G. Keller. Equilibrium states for s-unimodal maps. Ergodic Theory $\mathcal{E}$ Dynamical Systems, 18:765-789, 1998.

[25] P. Collet and C. Tresser. Ergodic theory and continuity of the Bowen-Ruelle measure for geometrical Lorenz flows. Fyzika, 20:33-48, 1988.

[26] W. de Melo and S. van Strien. One-dimensional dynamics. Springer Verlag, 1993.

[27] D. Dolgopyat. On decay of correlations in Anosov flows. Ann. of Math. (2), 147(2):357-390, 1998.

[28] D. Dolgopyat. Prevalence of rapid mixing in hyperbolic flows. Ergodic Theory Dynam. Systems, 18(5):1097-1114, 1998.

[29] R. S. Ellis. Entropy, large deviations, and statistical mechanics. Reprint of the 1985 original. Classics in Mathematics. Springer-Verlag, Berlin, 2006.

[30] M. Field, I. Melbourne, and A. Törok. Stability of mixing and rapid mixing for hyperbolic flows. Annals of Mathematics, to appear, 2007.

[31] J. M. Freitas. Continuity of SRB measure and entropy for Benedicks-Carleson quadratic maps. Nonlinearity, 18:831-854, 2005.

[32] S. Gouëzel. Decay of correlations for nonuniformly expanding systems. Bull. Soc. Math. France, 134(1):1-31, 2006.

[33] J. Guckenheimer. A strange, strange attractor. In The Hopf bifurcation theorem and its applications, pages 368-381. Springer Verlag, 1976.

[34] J. Guckenheimer and R. F. Williams. Structural stability of Lorenz attractors. Publ. Math. IHES, 50:59-72, 1979.

[35] F. Hofbauer and G. Keller. Quadratic maps without asymptotic measure. Comm. Math. Phys., 127:319-337, 1990. 
[36] G. Keller. Generalized bounded variation and applications to piecewise monotonic transformations. Z. Wahrsch. Verw. Gebiete, 69(3):461-478, 1985.

[37] Y. Kifer. Large deviations in dynamical systems and stochastic processes. Transactions of the Americal Mathematical Society, 321(2):505-524, 1990.

[38] Y. Kifer and S. E. Newhouse. A global volume lemma and applications. Israel J. Math., 74(2-3):209-223, 1991.

[39] F. Ledrappier. Some properties of absolutely continuous invariant measures on an interval. Ergod. Th. \& Dynam. Sys., 1:77-93, 1981.

[40] F. Ledrappier and L.-S. Young. The metric entropy of diffeomorphisms I. characterization of measures satisfying Pesin's entropy formula. Ann. of Math, 122:509-539, 1985.

[41] F. Ledrappier and L.-S. Young. The metric entropy of diffeomorphisms. II. Relations between entropy, exponents and dimension. Ann. of Math. (2), 122(3):540-574, 1985.

[42] P.-D. Liu. Pesin's Entropy Formula for endomorphisms. Nagoya Math. J., 150:197209, 1998.

[43] E. N. Lorenz. Deterministic nonperiodic flow. J. Atmosph. Sci., 20:130-141, 1963.

[44] S. Luzzatto, I. Melbourne, and F. Paccaut. The Lorenz attractor is mixing. Comm. Math. Phys., 260(2):393-401, 2005.

[45] I. Melbourne. Rapid decay of correlations for nonuniformly hyperbolic flows. Trans. Amer. Math. Soc., 359(5):2421-2441 (electronic), 2007.

[46] I. Melbourne and M. Nicol. Large deviations for nonuniformly hyperbolic systems. Preprint., 2006.

[47] K. Oliveira and M. Viana. Existence and uniqueness of maximizing measures for robust classes of local diffeomorphisms. Discrete and Continuous Dynamical Systems, 15(1):225-236, 2006.

[48] Y. Pesin and Y. Sinai. Gibbs measures for partially hyperbolic attractors. Ergod. Th. \& Dynam. Sys., 2:417-438, 1982.

[49] V. Pliss. On a conjecture due to Smale. Diff. Uravnenija, 8:262-268, 1972.

[50] R. C. Robinson. An introduction to dynamical systems: continuous and discrete. Pearson Prentice Hall, Upper Saddle River, NJ, 2004.

[51] D. Ruelle. The thermodynamical formalism for expanding maps. Comm. Math. Phys., 125:239-262, 1989. 
[52] D. Ruelle. Thermodynamic formalism. The mathematical structures of equilibrium statistical mechanics. Cambridge Mathematical Library. Cambridge University Press, Cambridge, 2nd edition edition, 2004.

[53] M. Rychlik. Bounded variation and invariant measures. Studia Math., 76:69-80, 1983.

[54] W. Tucker. The Lorenz attractor exists. C. R. Acad. Sci. Paris, 328, Série I:1197-1202, 1999.

[55] W. Tucker. A rigorous ode solver and smale's 14th problem. Found. Comput. Math., 2(1):53-117, 2002.

[56] M. Viana. Stochastic dynamics of deterministic systems. Publicações Matemáticas do IMPA. [IMPA Mathematical Publications]. Instituto de Matemática Pura e Aplicada (IMPA), Rio de Janeiro, 1997. 21 Colóquio Brasileiro de Matemática. [21th Brazilian Mathematics Colloquium].

[57] S. Waddington. Large deviations asymptotics for Anosov flows. Annales de l'Institut Henri Poincare, Section C, 13(4):445-484, 1996.

[58] L.-S. Young. Some large deviation results for dynamical systems. Trans. Amer. Math. Soc., 318(2):525-543, 1990.

[59] L.-S. Young. Statistical properties of dynamical systems with some hyperbolicity. Annals of Math., 147:585-650, 1998. 